\title{
Fitting mixtures of linear regressions
}

\author{
Susana Faria ${ }^{\mathrm{a} *}$ and Gilda Soromenho ${ }^{\mathrm{b}}$ \\ ${ }^{a}$ Department of Mathematics for Science and Technology, Research CMAT, University of Minho, \\ Guimarães, Portugal; ${ }^{b}$ Faculty of Psychology and Sciences of Education, Research LEAD, \\ University of Lisbon, Lisbon, Portugal
}

(Received 1 April 2008; final version received 30 October 2008)

\begin{abstract}
In most applications, the parameters of a mixture of linear regression models are estimated by maximum likelihood using the expectation maximization (EM) algorithm. In this article, we propose the comparison of three algorithms to compute maximum likelihood estimates of the parameters of these models: the EM algorithm, the classification EM algorithm and the stochastic EM algorithm. The comparison of the three procedures was done through a simulation study of the performance (computational effort, statistical properties of estimators and goodness of fit) of these approaches on simulated data sets.

Simulation results show that the choice of the approach depends essentially on the configuration of the true regression lines and the initialization of the algorithms.
\end{abstract}

Keywords: mixtures of linear regressions; maximum likelihood estimation; EM algorithm; classification EM algorithm; simulation study

\section{Introduction}

Finite mixture models have provided a mathematically based approach to the statistical modelling of a wide variety of random phenomena. Applications of mixture distributions can be found in various fields of statistical applications such as agriculture, biology, economics, medicine and genetics; see e.g. [1-3] for a review.

Within the family of mixture models, mixtures of linear regressions have also been studied extensively, especially when no information about membership of the points assigned to each line was available.

Mixtures of linear regression models were introduced by Quandt and Ramsey [4] as a very general form of 'switching regression'. They used a technique based on a moment-generating function to estimate the parameters. However, it has mainly been studied from a likelihood point of view. De Veaux [5] developed an EM approach to fit the two regression situations. Jones and McLachlan [6] applied mixtures of regressions in a data analysis and used the EM algorithm to fit these models. Turner [7] fitted a two-component mixture of one variable linear regression to

*Corresponding author. Email: sfaria@mct.uminho.pt

ISSN 0094-9655 print/ISSN 1563-5163 online

(C) 2010 Taylor \& Francis

DOI: $10.1080 / 00949650802590261$

http://www.informaworld.com 
a data set using the EM algorithm. Hawkins et al. [8] studied the problem of determining the number of components in a mixture of linear regression models using methods derived from the likelihood equation. More recently, Zhu and Zhang [9] established asymptotic theory for maximum likelihood estimators in mixture regression models.

In this article, we study the procedure for fitting mixtures of linear regressions by means of maximum likelihood. We apply three maximization algorithms to obtain the maximum likelihood estimates: the expectation maximization (EM) algorithm (see [10]), the classification EM (CEM) algorithm (see [11]) and the stochastic EM (SEM) algorithm (see [12]).

The comparison of EM and CEM approaches in a cluster analysis is well known in the mixture models literature. Under the Gaussian mixture, Ganesalingam [13] has performed numerical experiments to compare the two approaches in practical situations. An extension of this study was performed by Celeux and Govaert [14], in order to specify the influence of the sample sizes and the dependence of the used algorithms over their initial values. Considering the case of binary data, Govaert and Nadif [15] presented an extension of the comparisons to Bernoulli models.

Some comparisons of EM and SEM approaches in a mixture of distributions are also available. Celeux et al. [16] have investigated the practical behaviour of these algorithms through intensive Monte Carlo numerical simulations and a real data study. Dias and Wedel [17] have compared EM and SEM algorithms to estimate the parameters of Gaussian mixture model.

Our goal is to compare the performance of these three approaches on mixtures of linear regressions. A simulation study is designed to investigate this problem.

The article is organized as follows: in Section 2, we present the mixture of linear regression model and the three maximization algorithms to obtain the maximum likelihood estimates. Section 3 provides a simulation study investigating the performance of the algorithms for fitting two- and three-component mixtures of linear regression models. In Section 4, the conclusions of our study are drawn and additional comments are given.

\section{Fitting mixtures of linear regressions}

The mixture of linear regression model is given as follows:

$$
y_{i}=\left\{\begin{array}{cc}
x_{i}^{\mathrm{T}} \beta_{1}+\epsilon_{i 1} & \text { with probability } \pi_{1}, \\
x_{i}^{\mathrm{T}} \beta_{2}+\epsilon_{i 2} & \text { with probability } \pi_{2}, \\
\vdots & \text { with probability } \pi_{J}
\end{array}\right.
$$

where $y_{i}$ is the value of the response variable in the $i$ th observation; $x_{i}^{\mathrm{T}}(i=1, \ldots, n)$ denotes the transpose of the $(\mathrm{p}+1)$-dimensional vector of independent variables for the $i$ th observation, $\beta_{j}(j=1, \ldots, J)$ denotes the $(\mathrm{p}+1)$-dimensional vector of regressor variables for the $j$ th component, $\pi_{j}$ are the mixing probabilities $\left(0<\pi_{j}<1\right.$, for all $j=1, \ldots, J$ and $\left.\sum_{j} \pi_{j}=1\right)$. Finally, $\epsilon_{i j}$ are the random errors; under the assumption of normality, we have $\epsilon_{i j} \sim N\left(0, \sigma_{j}^{2}\right)(i=$ $1, \ldots, n ; j=1, \ldots, J)$.

Given a set of independent observations $y_{1}, y_{2}, \ldots, y_{n}$, corresponding to values $x_{1}, x_{2}, \ldots, x_{n}$ of the predictor $x$, the complete parameter set of the mixture model, $\theta=$ $\left(\pi_{1}, \ldots, \pi_{J}, \beta_{1}, \ldots, \beta_{J}, \sigma_{1}^{2}, \ldots, \sigma_{J}^{2}\right)$, can be estimated by maximizing the log-likelihood

$$
L\left(\theta \mid x_{1}, \ldots, x_{n}, y_{1}, \ldots, y_{n}\right)=\sum_{i=1}^{n} \log \left(\sum_{j=1}^{J} \pi_{j} \phi_{j}\left(y_{i} \mid x_{i}\right)\right),
$$


where $\phi_{j}\left(y_{i} \mid x_{i}\right)$ denotes the density of an univariate Gaussian distribution with mean $x_{i}^{\mathrm{T}} \beta_{j}$ and variance $\sigma_{j}^{2}$.

\subsection{The EM algorithm}

The standard tool for finding the maximum likelihood solution is the EM algorithm (see [10] and [3]). The EM algorithm is a broadly applicable approach to the iterative computation of maximum likelihood estimates when the observations can be viewed as incomplete data. The idea here is to think of the data as consisting of triples $\left(x_{i}, y_{i}, z_{i}\right)$, where $z_{i}$ is the unobserved indicator that specifies the mixture component from which the observation $y_{i}$ is drawn.

The EM algorithm is easy to program and proceeds iteratively in two steps, $E$ (for expectation) and $M$ (for maximization).

Let $\theta^{(r)}$ be the estimate of the parameters after the $r$ th iteration. On the $(r+1)$ th iteration, the $E$-step of the EM algorithm involves the calculation of the $Q$-function, which is the expectation of the complete-data log-likelihood conditional on the current parameter estimates and the observed data,

$$
Q\left(\theta, \theta^{(r)}\right)=\sum_{i=1}^{n} \sum_{j=1}^{J} w_{i j}^{(r)} \phi_{j}\left(y_{i} \mid x_{i}\right)
$$

where

$$
w_{i j}^{(r)}=\frac{\pi_{j}^{(r)} \phi_{j}\left(y_{i} \mid x_{i}\right)}{\sum_{j=1}^{J} \pi_{j}^{(r)} \phi_{j}\left(y_{i} \mid x_{i}\right)} \quad(i=1, \ldots, n ; j=1, \ldots, J)
$$

is the estimate of the posterior probability that the $i$ th observation belongs to the $j$ th component of the mixture after the $r$ th iteration.

The $M$-step updates the estimate $\theta^{(r+1)}$ that maximizes the $Q$-function with respect to $\theta$. It is equivalent to computing the sample proportion and the weighted least-squares estimates when performing a weighted regression of $y_{1}, \ldots, y_{n}$ on $x_{1}, \ldots, x_{n}$ with weights $w_{1 j}, \ldots, w_{n j}(j=$ $1, \ldots, J)$.

It follows that on the $M$-step of the $(r+1)$ th iteration, the current fit for the parameters is given explicitly by

$$
\begin{aligned}
\hat{\pi}_{j}^{(r+1)} & =\frac{\sum_{i=1}^{n} w_{i j}^{(r)}}{n} \quad(j=1, \ldots, J) \\
\hat{\beta}_{j}^{(r+1)} & =\left(X^{\mathrm{T}} W_{j} X\right)^{-1} X^{\mathrm{T}} W_{j} Y \quad(j=1, \ldots, J),
\end{aligned}
$$

where $X$ is a $n \times(p+1)$ matrix of predictors, $W_{j}$ is a $n \times n$ diagonal matrix with diagonal entries $w_{i j}^{(r)}$ and $Y$ is a $n \times 1$ vector of response variable; and

$$
\hat{\sigma}_{j}^{2(r+1)}=\frac{\sum_{i=1}^{n} w_{i j}^{(r)}\left(y_{i}-x_{i}^{\mathrm{T}} \hat{\beta}_{j}^{(r+1)}\right)^{2}}{\sum_{i=1}^{n} w_{i j}^{(r)}} \quad(j=1, \ldots, J) .
$$

The $E$ - and $M$ - steps are alternated repeatedly until some specified convergence criterion is achieved.

The characteristics of the EM algorithm are well documented (see for instance [3]). 


\subsection{The CEM algorithm}

To fit mixtures of linear regressions, we also make use of a classification version of the EM algorithm, the so-called CEM algorithm. The CEM algorithm maximizes in $\theta$ and $z_{1}, \ldots, z_{n}$ the complete data classification log-likelihood, where the missing component label $z_{i}$ of each sample observation is included in the data set:

$$
\mathrm{CL}\left(\theta \mid z_{1}, \ldots, z_{n}, x_{1}, \ldots, x_{n}, y_{1}, \ldots, y_{n}\right)=\sum_{j=1}^{J} \sum_{\left\{i \mid z_{i}=j\right\}} \log \left(\pi_{j} \phi_{j}\left(y_{i} \mid x_{i}\right)\right),
$$

where $\left\{i \mid z_{i}=j\right\}$ is the set of observations arising from the $j$ th mixture component.

The CEM algorithm incorporates a classification step (C-step) between the E- and M-steps of EM. This classification step involves assigning each observation to one of the $J$ components that provides the largest posterior probability $w_{i j}$.

Thus, an iteration of CEM algorithm consists of three steps. The E-step of the CEM algorithm is identical to the E-step of the EM algorithm.

On the C-step of the $(r+1)$ th iteration, a partition $P^{(r+1)}=\left(P_{1}^{(r+1)}, \ldots, P_{J}^{(r+1)}\right)$ of $\left(x_{1}, y_{1}\right), \ldots,\left(x_{n}, y_{n}\right)$ is designed by assigning each observation to the component for which $w_{i j}^{(r)}$ is largest (if the maximum posterior probability is not unique, we choose the component with the smallest index). We have,

$$
P_{j}^{(r+1)}=\left\{\left(x_{i}, y_{i}\right): w_{i j}^{(r)}=\arg _{h} \max w_{i h}^{(r)}\right\}
$$

if $w_{i j}^{(r)}=w_{i h}^{(r)}$ and $j<h$ then $\left(x_{i}, y_{i}\right) \in P_{j}^{(r+1)}(j=1, \ldots, J)$. If one of the $P^{(r+1)}$ is empty or has only one observation, it must be considered that the mixture has $J-1$ components instead of $J$ and the estimation process begins with $J-1$ components.

The M-step updates the estimate $\theta^{(r+1)}$ using the sub-samples $P_{j}^{(r+1)}$. It follows that on the M-step of the $(r+1)$ th iteration, the current fit for the parameters is given explicitly by

$$
\hat{\pi}_{j}^{(r+1)}=\frac{n_{j}}{n} \quad(j=1, \ldots, J),
$$

where $n_{j}$ is the total number of observations arising from component $j$;

$$
\hat{\beta}_{j}^{(r+1)}=\left(X_{j}^{\mathrm{T}} W_{j} X_{j}\right)^{-1} X_{j}^{\mathrm{T}} W_{j} Y_{j} \quad(j=1, \ldots, J),
$$

where $X_{j}$ is a $n_{j} \times(p+1)$ matrix of predictors for the $j$ th component, $W_{j}$ is a $n_{j} \times n_{j}$ diagonal matrix with diagonal entries $w_{i j}^{(r)}$ and $Y_{j}$ is a $n_{j} \times 1$ vector of response variable for the $j$ th component; and

$$
\hat{\sigma}_{j}^{2(r+1)}=\frac{\sum_{i=1}^{n_{j}} w_{i j}^{(r)}\left(y_{i}-x_{i}^{\mathrm{T}} \beta_{j}^{(r+1)}\right)^{2}}{\sum_{i=1}^{n_{j}} w_{i j}^{(r)}} .
$$

The E-, C- and M-steps are alternated repeatedly until some specified convergence criterion is achieved.

CEM algorithm is a K-means-like algorithm and contrary to EM, it converges in a finite number of iterations.

\subsection{The SEM algorithm}

We also apply a procedure for fitting mixtures of linear regressions using a stochastic version of the EM algorithm, the so-called SEM algorithm. The SEM algorithm incorporates a stochastic 
step (S-step) between the E- and M-steps of EM. This stochastic step simulates a realization of the unobserved indicator $z_{i}, i=1, \ldots, n$ by drawing them at random from their current conditional distribution.

Thus, an iteration of SEM algorithm consists of three steps. The E-step of the SEM algorithm is identical to the E-step of the EM algorithm.

On the S-step of the $(r+1)$ th iteration, a partition $P^{(r+1)}=\left(P_{1}^{(r+1)}, \ldots, P_{J}^{(r+1)}\right)$ of $\left(x_{1}, y_{1}\right), \ldots,\left(x_{n}, y_{n}\right)$ is designed by assigning each observation at random to one of the mixture components according to the multinomial distribution with parameter $w_{i j}^{(r)}$, given by Equation (4). The M-step of the SEM algorithm is identical to the M-step of the CEM algorithm.

SEM does not converge pointwise. It generates a Markov chain whose stationary distribution is more or less concentrated around the maximum likelihood parameter estimate.

\section{Simulation study of algorithm performance}

A simulation study was performed to assess the performance of the maximum likelihood estimates obtained via the EM algorithm, the CEM algorithm and the SEM algorithm. Data were simulated under a two to three component mixture of linear regressions. We used the freeware $R$ (see [18]) to develop the simulation program.

\subsection{Design of the study}

Initial conditions. In our simulation study, two different strategies of choosing initial values were considered. In the first strategy, the true values were used as the starting values. In the other strategy we ran the algorithm 20 times from random initial position and selected the solution out of 20 runs which provided the best value of the optimized criterion (see [14]).

Stopping rules. A rather strict stopping criterion for the EM and the CEM algorithms was used: iterations were stopped when the relative change in log-likelihood between two successive iterations were less than $10^{-10}$. The stopping rule for the SEM algorithm was the total number of iterations required for convergence by the EM algorithm. We do not use the same stopping criteria because the slow convergence of the SEM algorithm makes such criteria hazardous.

Number of samples. For each type of simulated data set, 200 samples of size $n$ were simulated.

Configurations of the true regression lines. We considered two typical configurations of the true regression lines: parallel and concurrent. These configurations are expected to affect the performance of the proposed algorithms.

Data set. Each datum $\left(x_{i}, y_{i}\right)$ was generated by the following scheme. First, a uniform $[0,1]$ random number $c_{i}$ was generated and its value was used to select a particular component $j$ from mixture of regression models. Next, $x_{i}$ was randomly generated from a uniform $\left[x_{L}, x_{U}\right]$ distribution and a normal random variate $\epsilon_{j i}$ with mean 0 and variance $\sigma_{j}^{2}$ was calculated. Finally, the value $y_{i}$ was assigned using $x_{i}, \epsilon_{j i}$ and the appropriate model parameters (see [19]). We have chosen $x_{L}=-1$ and $x_{U}=3$ and in previous simulation studies we have obtained the same simulation results when these values were changed.

Measures of algorithm performance: In order to examine the performance of two algorithms, the following criteria were used:

- the mean number of iterations required for convergence (which gives an indication about the computing time needed),

- the statistical properties of the estimators $\theta$ : 
(i) bias of the parameter estimates over the 200 replications:

$$
\operatorname{BIAS}\left(\hat{\theta}_{j}\right)=\frac{1}{200} \sum_{m=1}^{200} \hat{\theta}_{j}^{(m)}-\theta_{j},
$$

where $\theta_{j}=\left(\pi_{j}, \beta_{j}, \sigma_{j}^{2}\right)$ and $\hat{\theta}_{j}^{(m)}=\left(\hat{\pi}_{j}^{(m)}, \hat{\beta}_{j}^{(m)}, \hat{\sigma}_{j}^{2(m)}\right), j=1, \ldots, J$ of the $m$ th replication with $m=1, \ldots, 200$.

(ii) the mean square error (MSE) of the parameter estimates over the 200 replications:

$$
\operatorname{MSE}\left(\hat{\theta}_{j}\right)=\frac{1}{200} \sum_{m=1}^{200}\left(\hat{\theta}_{j}^{(m)}-\theta_{j}\right)^{2}
$$

- the root mean-squared error of prediction (MRSEP):

$$
\operatorname{MRSEP}=\frac{1}{200} \sum_{m=1}^{200} \operatorname{RMSEP}^{(m)},
$$

where RMSEP ${ }^{(m)}$ is the root mean-squared error of prediction of the $m$ th replication based on $K$-fold cross-validation, which is given by

$$
\operatorname{RMSEP}^{(m)}=\sqrt{\frac{1}{n} \sum_{i=1}^{n}\left(y_{i}-\hat{y}_{i}^{(m)}\right)^{2}}
$$

with $\hat{y}_{i}^{(m)}$ corresponding to the fitted value of the observation $i$ of the $m$ th replication.

For the $K$-fold cross-validation, we have chosen $K=5$ and $K=10$ (see [20], Chapter 7).

The simulation process consists of the following steps:

(1) Create a data set of size $n$.

(2) Fit a mixture of linear regression models to the data using the EM, the CEM and the SEM algorithms. Save the number of iterations required for convergence and the estimated parameters $\hat{\theta}=\left(\hat{\pi}_{1}, \ldots, \hat{\pi}_{J}, \hat{\beta}_{1}, \ldots, \hat{\beta}_{J}, \hat{\sigma}_{1}^{2}, \ldots, \hat{\sigma}_{J}^{2}\right)$.

(3) Split the data into $K$ roughly equal-sized parts. For the $k$ th part, fit the model to the other $K-1$ parts of the data using the EM, the CEM and the SEM algorithms, and calculate the prediction error of the fitted model when predicting the $k$ th part of the data. Do this for $k=1, \ldots, K$, combine the $K$ estimates of prediction error and compute the corresponding value for RMSEP.

(4) Repeat steps 1-3, for a total of 200 trials. Compute the mean number of iterations required for convergence, the bias of the parameter estimates $\left(\operatorname{BIAS}\left(\hat{\theta}_{j}\right)\right)$, the mean square error $\left(\operatorname{MSE}\left(\hat{\theta}_{j}\right)\right)$ of the parameter estimates and the root mean-squared error of prediction (MRSEP).

\subsection{Simulation results: two component mixtures of linear regressions}

For two component models $(J=2)$, samples of three different sizes $n(n=50,100,500)$ were generated for each set of true parameter values $(\beta, \sigma)$ shown on Table 1 and the mixing proportion $\pi_{1}$ lying from 0.1 to 0.9 . For instance, we present in Figure 1 typical scatter plots for samples with size 100 and mixing proportion 0.5 .

Tables 2 and 3 provide the mean number of iterations required for convergence using the EM and the CEM algorithms for fitting two-component mixtures of linear regression models. In all 
Table 1. True parameter values for the essays with a two-component mixture of linear regressions.

\begin{tabular}{lcccccc}
\hline Configuration & $\beta_{10}$ & $\beta_{20}$ & $\beta_{11}$ & $\beta_{21}$ & $\sigma_{1}^{2}$ & $\sigma_{2}^{2}$ \\
\hline Parallel & 0 & 4 & 1 & 1 & $1^{2}$ & $1^{2}$ \\
Concurrent & 1 & 0 & -1 & 0.5 & $0.2^{2}$ & $0.2^{2}$ \\
\hline
\end{tabular}

Parallel

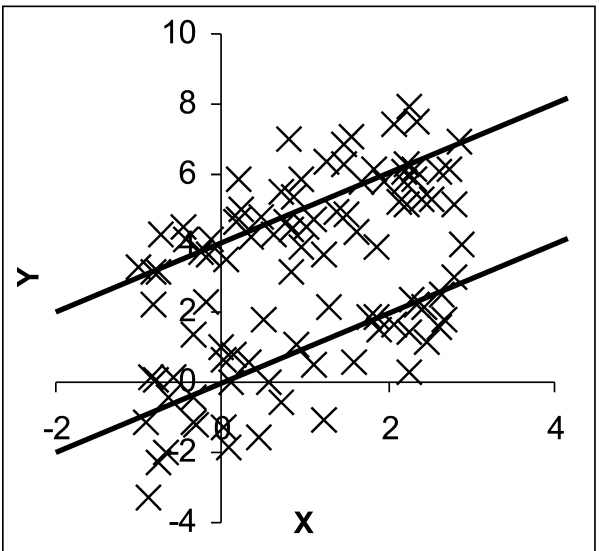

Concurrent

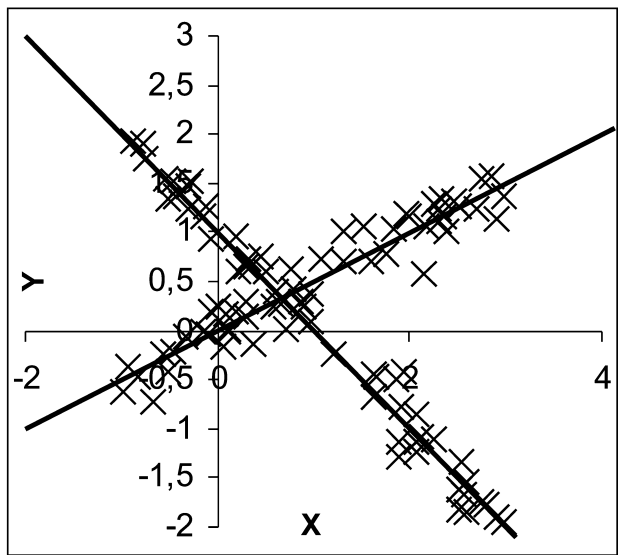

Figure 1. Scatter plot of samples from two-component models with $n=100$ and $\pi=(0.5 ; 0.5)$.

the cases, the mean number of iterations for convergence is smaller using the CEM algorithm rather than using the EM algorithm.

Tables 4-7 provide the MSE and the bias of the parameter estimates over the 200 replications of the two-component mixtures of linear regression models, when the mixing proportion $\pi_{1}$ is chosen to be $0.2,0.5$ and 0.7 .

When the true regression lines are parallel and the algorithms are initiated with the true parameter values, Table 4 shows that CEM estimates have smaller MSE than EM and SEM estimates. It is evident that the estimates obtained by the three algorithms have relatively small bias and MSE tends to decrease as the sample size increases.

Table 2. The mean number of iterations required for convergence using the EM and CEM algorithms for two-component mixtures of linear regressions when the true values were used as the starting values.

\begin{tabular}{|c|c|c|c|c|c|c|c|c|c|c|c|c|}
\hline \multirow[b]{3}{*}{$\pi_{1}$} & \multicolumn{6}{|c|}{ Parallel } & \multicolumn{6}{|c|}{ Concurrent } \\
\hline & \multicolumn{2}{|c|}{$n=50$} & \multicolumn{2}{|c|}{$n=100$} & \multicolumn{2}{|c|}{$n=500$} & \multicolumn{2}{|c|}{$n=50$} & \multicolumn{2}{|c|}{$n=100$} & \multicolumn{2}{|c|}{$n=500$} \\
\hline & EM & CEM & EM & CEM & EM & CEM & EM & CEM & EM & CEM & EM & CEM \\
\hline 0.1 & 31.40 & 8.47 & 37.77 & 10.18 & 32.36 & 11.90 & 13.78 & 8.23 & 10.72 & 7.25 & 8.44 & 7.28 \\
\hline 0.2 & 35.45 & 9.61 & 38.22 & 10.95 & 26.46 & 12.33 & 10.98 & 7.76 & 9.40 & 7.67 & 7.21 & 7.02 \\
\hline 0.3 & 40.02 & 11.05 & 33.20 & 11.49 & 24.02 & 12.26 & 10.92 & 8.38 & 8.46 & 7.71 & 7.46 & 7.26 \\
\hline 0.4 & 32.52 & 10.62 & 28.40 & 10.90 & 23.93 & 11.68 & 9.13 & 8.07 & 8.44 & 7.73 & 7.99 & 7.36 \\
\hline 0.5 & 34.19 & 10.77 & 30.03 & 11.07 & 23.14 & 10.79 & 9.16 & 8.15 & 8.07 & 7.96 & 7.85 & 7.65 \\
\hline 0.6 & 33.63 & 10.38 & 31.40 & 11.40 & 23.27 & 11.86 & 9.73 & 8.17 & 8.51 & 8.07 & 7.98 & 7.50 \\
\hline 0.7 & 34.00 & 10.24 & 37.37 & 11.20 & 23.94 & 12.08 & 10.28 & 7.81 & 8.24 & 7.89 & 7.65 & 7.37 \\
\hline 0.8 & 33.60 & 9.66 & 35.51 & 10.75 & 26.42 & 12.41 & 10.38 & 7.32 & 9.11 & 7.49 & 7.46 & 7.11 \\
\hline 0.9 & 24.09 & 8.06 & 39.72 & 10.04 & 36.74 & 12.00 & 12.50 & 7.43 & 10.70 & 7.31 & 8.17 & 7.07 \\
\hline
\end{tabular}


Table 3. The mean number of iterations required for convergence using the EM and CEM algorithms for two-component mixtures of linear regressions when the algorithms were initiated by random numbers (second strategy).

\begin{tabular}{|c|c|c|c|c|c|c|c|c|c|c|c|c|}
\hline \multirow[b]{3}{*}{$\pi_{1}$} & \multicolumn{6}{|c|}{ Parallel } & \multicolumn{6}{|c|}{ Concurrent } \\
\hline & \multicolumn{2}{|c|}{$n=50$} & \multicolumn{2}{|c|}{$n=100$} & \multicolumn{2}{|c|}{$n=500$} & \multicolumn{2}{|c|}{$n=50$} & \multicolumn{2}{|c|}{$n=100$} & \multicolumn{2}{|c|}{$n=500$} \\
\hline & EM & CEM & EM & CEM & EM & CEM & EM & CEM & EM & CEM & EM & CEM \\
\hline 0.1 & 77.97 & 20.31 & 91.77 & 20.66 & 100.08 & 28.15 & 25.10 & 15.82 & 20.51 & 14.52 & 17.33 & 12.84 \\
\hline 0.2 & 68.92 & 19.57 & 80.57 & 21.52 & 68.67 & 27.42 & 19.54 & 15.43 & 17.27 & 15.55 & 14.98 & 11.46 \\
\hline 0.3 & 71.79 & 20.16 & 70.18 & 21.67 & 57.12 & 28.25 & 18.81 & 14.93 & 17.06 & 16.94 & 15.24 & 10.22 \\
\hline 0.4 & 79.85 & 19.22 & 91.99 & 21.08 & 108.41 & 26.81 & 17.14 & 15.91 & 16.38 & 16.27 & 14.91 & 12.94 \\
\hline 0.5 & 85.32 & 18.74 & 134.24 & 21.71 & 204.66 & 25.00 & 15.75 & 15.69 & 17.49 & 16.56 & 13.53 & 10.74 \\
\hline 0.6 & 80.65 & 18.39 & 101.81 & 20.66 & 106.72 & 25.94 & 16.14 & 15.42 & 17.71 & 16.74 & 13.69 & 11.09 \\
\hline 0.7 & 75.97 & 19.52 & 81.65 & 20.74 & 54.29 & 26.40 & 17.67 & 17.38 & 17.86 & 17.35 & 13.85 & 11.07 \\
\hline 0.8 & 71.41 & 19.99 & 78.75 & 20.92 & 68.68 & 26.69 & 17.98 & 17.08 & 17.82 & 17.36 & 14.56 & 12.76 \\
\hline 0.9 & 66.57 & 19.71 & 102.90 & 20.98 & 98.65 & 27.85 & 22.97 & 18.03 & 20.46 & 18.11 & 16.63 & 14.60 \\
\hline
\end{tabular}

When the true regression lines are parallel but the initialization of the algorithms is made by random numbers (second strategy), Table 5 shows that SEM performs better than EM and CEM. In this case, however the performance of all three algorithms decrease, producing estimates that have higher MSE and bias.

When the true regression lines are concurrent and the algorithms are initiated with the true parameter values, Table 6 shows that the three algorithms have practically the same behaviour. Also, the MSE of the parameter estimates decreases whenever the sample size increases.

When the true regression lines are concurrent but the algorithms are initiated by random numbers (second strategy), Table 7 shows that CEM estimates of the parameters have higher MSE and bias than EM and SEM estimates. In generality, EM outperforms SEM by producing estimates of the parameters that have smaller MSE and bias.

The resulting values of MRSEP based on 10-fold cross-validation when the true values were used as the starting values for each of the configurations of the true regression lines are plotted in Figure 2. Similar results were obtained calculating MRSEP based on 5-fold cross-validation. When the true regression lines are parallel, the CEM algorithm performs generally better, however, for a sample size of 500 and when the mixing proportions are equal, it seems that the three algorithms have practically the same behaviour. When the true regression lines are concurrent, it seems that the three algorithms have practically the same behaviour. However, for a sample size of 50 and 100 , the performances of the CEM and SEM algorithms decrease when the mixing proportion is smaller and the EM algorithm performs better in those cases.

The resulting values of MRSEP based on 10-fold cross-validation when the second strategy was used as the starting values for each of the configurations of the true regression lines are plotted in Figure 3. Similar results were obtained calculating MRSEP based on 5-fold cross-validation. When the true regression lines are parallel, the SEM algorithm performs generally better; however, for a sample size of 500, it seems that the EM algorithm performs better. Figure 3 suggests that the EM algorithm performs always better in fitting a two-component mixture of linear regressions when the true regression lines are concurrent and the second strategy was used as the starting value.

\subsection{Simulation results: three-component mixtures of linear regressions}

For three component models $(J=3)$, samples of size $n=100$ and $n=500$ were generated for the two sets of parameter values $(\beta, \sigma)$ shown in Table 8 . For illustration we show scatter plots of random samples of 100 points in Figure 4. 
Table 4. Mean square error and bias of estimates based on 200 replications of the two-component mixtures of linear regression models when the true regression lines are parallel and the true values are used as the starting values.

\begin{tabular}{|c|c|c|c|c|c|c|c|c|c|c|c|}
\hline$n$ & $\pi_{1}$ & Algorithm & & $\beta_{10}$ & $\beta_{11}$ & $\beta_{20}$ & $\beta_{21}$ & $\sigma_{1}^{2}$ & $\sigma_{2}^{2}$ & $\pi_{1}$ & $\pi_{2}$ \\
\hline \multirow[t]{18}{*}{50} & \multirow[t]{6}{*}{0.2} & \multirow[t]{2}{*}{ EM } & BIAS & 0.1082 & -0.0047 & 0.0031 & 0.0123 & -0.1532 & -0.0583 & 0.0139 & -0.0139 \\
\hline & & & MSE & 0.6550 & 0.2340 & 0.0690 & 0.0240 & 0.1428 & 0.0240 & 0.0064 & 0.0064 \\
\hline & & \multirow[t]{2}{*}{ CEM } & BIAS & -0.0939 & 0.0007 & -0.0085 & 0.0110 & -0.2571 & -0.0569 & -0.0049 & 0.0049 \\
\hline & & & MSE & 0.3166 & 0.1296 & 0.0526 & 0.0214 & 0.1175 & 0.0188 & 0.0030 & 0.0030 \\
\hline & & \multirow[t]{2}{*}{ SEM } & BIAS & -0.1407 & 0.0012 & -0.0284 & 0.0096 & -0.3109 & -0.0382 & -0.0059 & 0.0059 \\
\hline & & & MSE & 0.4272 & 0.1540 & 0.0788 & 0.0275 & 0.1820 & 0.0358 & 0.0077 & 0.0077 \\
\hline & \multirow[t]{6}{*}{0.5} & \multirow[t]{2}{*}{ EM } & BIAS & -0.0448 & 0.0154 & -0.0457 & 0.0022 & -0.0806 & -0.0298 & -0.0080 & 0.0080 \\
\hline & & & MSE & 0.1426 & 0.0475 & 0.1848 & 0.0461 & 0.0488 & 0.0625 & 0.0082 & 0.0082 \\
\hline & & \multirow[t]{2}{*}{ CEM } & BIAS & -0.0649 & 0.0096 & 0.0221 & 0.0047 & -0.1134 & -0.0870 & -0.0014 & 0.0014 \\
\hline & & & MSE & 0.0899 & 0.0350 & 0.0988 & 0.0359 & 0.0355 & 0.0353 & 0.0057 & 0.0057 \\
\hline & & \multirow[t]{2}{*}{ SEM } & BIAS & -0.0653 & 0.0131 & 0.0358 & 0.0070 & -0.1090 & -0.1040 & 0.0005 & -0.0005 \\
\hline & & & MSE & 0.1590 & 0.0444 & 0.1580 & 0.0447 & 0.0690 & 0.0669 & 0.0111 & 0.0111 \\
\hline & \multirow[t]{6}{*}{0.7} & \multirow[t]{2}{*}{ EM } & BIAS & 0.0197 & -0.0153 & -0.0069 & -0.0120 & -0.0425 & -0.1245 & -0.0032 & 0.0032 \\
\hline & & & MSE & 0.0887 & 0.0315 & 0.2785 & 0.0969 & 0.0289 & 0.1095 & 0.0066 & 0.0066 \\
\hline & & \multirow{2}{*}{ CEM } & BIAS & 0.0125 & -0.0092 & 0.1101 & 0.0030 & -0.0514 & -0.2074 & 0.0101 & -0.0101 \\
\hline & & & MSE & 0.0613 & 0.0263 & 0.1222 & 0.0584 & 0.0176 & 0.0815 & 0.0042 & 0.0042 \\
\hline & & \multirow[t]{2}{*}{ SEM } & BIAS & 0.0268 & -0.0166 & 0.1593 & -0.0136 & -0.0408 & -0.2379 & 0.0118 & -0.0118 \\
\hline & & & MSE & 0.1001 & 0.0322 & 0.2500 & 0.0802 & 0.0412 & 0.1245 & 0.0077 & 0.0077 \\
\hline \multirow[t]{18}{*}{100} & \multirow[t]{6}{*}{0.2} & \multirow[t]{2}{*}{ EM } & BIAS & 0.0524 & 0.0019 & 0.0128 & -0.0090 & -0.0786 & -0.0123 & 0.0063 & -0.0063 \\
\hline & & & MSE & 0.2358 & 0.0824 & 0.0341 & 0.0120 & 0.0832 & 0.0140 & 0.0029 & 0.0029 \\
\hline & & CEM & BIAS & -0.1081 & -0.0104 & 0.0082 & -0.0069 & -0.1986 & -0.0196 & -0.0055 & 0.0055 \\
\hline & & & MSE & 0.1336 & 0.0532 & 0.0294 & 0.0113 & 0.0686 & 0.0099 & 0.0019 & 0.0019 \\
\hline & & SEM & BIAS & -0.1569 & -0.0081 & 0.0054 & -0.0089 & -0.2263 & -0.0122 & -0.0088 & 0.0088 \\
\hline & & & MSE & 0.2016 & 0.0615 & 67 & 0.0123 & 0.0908 & 0.0160 & 0.0026 & 0.0026 \\
\hline & 0.5 & EM & BIAS & -0.0078 & 0.0051 & -0.0303 & 0.0148 & -0.0184 & -0.0389 & -0.0013 & 0.0013 \\
\hline & & & MSE & 0.0579 & 0.0210 & 0.0590 & 0.0200 & 0.0243 & 0.0211 & 0.0037 & 0.0037 \\
\hline & & CEM & BIAS & -0.0578 & 0.0053 & 0.0154 & 0.0137 & -0.0711 & -0.0885 & -0.0020 & 0.0020 \\
\hline & & & MSE & 0.0460 & 0.0177 & & 0.0174 & 0.0202 & 0.0205 & & 0.0034 \\
\hline & & SEM & BIAS & -0.0726 & 0.0063 & 0.0191 & 0.0170 & -0.0781 & -0.0914 & -0.0030 & 0.0030 \\
\hline & & & MSE & 0.0622 & 0.0202 & 0.0 & 0.0188 & 0.0253 & 0.0264 & 0.0043 & 0.0043 \\
\hline & 0.7 & EM & BIAS & -0.0248 & 0.0118 & -0.0695 & 0.0111 & -0.0327 & -0.0277 & -0.0131 & 0.0131 \\
\hline & & & MSE & 0.0381 & 0.0132 & 0.1602 & 0.0479 & 0.0138 & 0.0559 & 0.0048 & 0.0048 \\
\hline & & CEM & BIAS & -0.0206 & 0.0068 & 0.0773 & 0.0060 & -0.0453 & -0.1346 & 0.0003 & -0.0003 \\
\hline & & & MSE & 0.0307 & 0.0124 & 0.0799 & 0.0314 & 0.0101 & 0.0362 & 0.0027 & 0.0027 \\
\hline & & SEM & BIAS & -0.0363 & 0.0113 & 0.0729 & 0.0178 & -0.0516 & -0.1380 & -0.0017 & 0.0017 \\
\hline & & & MSE & 0.0388 & 0.0127 & 0.1019 & 0.0341 & 0.0145 & 0.0466 & 0.0035 & 0.0035 \\
\hline 500 & 0.2 & EM & BIAS & -0.0023 & 0.0150 & 0.0053 & -0.0007 & -0.0149 & -0.0 & & -0.0027 \\
\hline & & & MSE & 0.0337 & 0.0110 & 0.0053 & 0.0020 & 0.0101 & 0.0018 & 0.0004 & 0.0004 \\
\hline & & CEM & BIAS & -0.1456 & 0.0125 & 0.0081 & -0.0008 & -0.1360 & -0.0186 & -0.0051 & 0.0051 \\
\hline & & & MSE & 0.0421 & 0.0089 & 0.0053 & 0.0020 & 0.0237 & 0.0021 & 0.0004 & 0.0004 \\
\hline & & SEM & BIAS & -0.1654 & 0.0124 & 0.0151 & -0.0012 & -0.1413 & -0.0227 & -0.0052 & 0.0052 \\
\hline & & & MSE & 0.0496 & 0.0091 & 0.0058 & 0.0021 & 0.0253 & 0.0024 & 0.0004 & 0.0004 \\
\hline & 0.5 & EM & BIAS & 0.0039 & -0.0001 & -0.0058 & 0.0006 & -0.0131 & -0.0046 & -0.0009 & 0.0009 \\
\hline & & & MSE & 0.0101 & 0.0037 & 0.0098 & 0.0036 & 0.0041 & 0.0031 & 0.0005 & 0.0005 \\
\hline & & CEM & BIAS & -0.0442 & 0.0001 & 0.0427 & 0.0010 & -0.0671 & -0.0594 & -0.0008 & 0.0008 \\
\hline & & & MSE & 0.0106 & 0.0035 & 0.0105 & 0.0035 & 0.0073 & 0.0059 & 0.0005 & 0.0005 \\
\hline & & SEM & BIAS & -0.0553 & -0.0005 & 0.0555 & 0.0018 & -0.0716 & -0.0655 & -0.0003 & 0.0003 \\
\hline & & & MSE & 0.0117 & 0.0035 & 0.0128 & 0.0036 & 0.0082 & 0.0068 & 0.0006 & 0.0006 \\
\hline & 0.7 & EM & BIAS & 0.0044 & 0.0001 & -0.0065 & 0.0075 & -0.0067 & -0.0106 & -0.0001 & 0.0001 \\
\hline & & & MSE & 0.0065 & 0.0025 & 0.0165 & 0.0065 & 0.0023 & 0.0068 & 0.0005 & 0.0005 \\
\hline & & CEM & BIAS & -0.0134 & -0.0002 & 0.0897 & 0.0074 & -0.0338 & -0.1008 & 0.0046 & -0.0046 \\
\hline & & & MSE & 0.0064 & 0.0024 & 0.0211 & 0.0056 & 0.0032 & 0.0142 & 0.0005 & 0.0005 \\
\hline & & SEM & BIAS & -0.0209 & 0.0004 & 0.1121 & 0.0073 & -0.0367 & -0.1091 & 0.0057 & -0.0057 \\
\hline & & & MSE & 0.0071 & 0.0024 & 0.0260 & 0.0056 & 0.0036 & 0.0164 & 0.0005 & 0.0005 \\
\hline
\end{tabular}


Table 5. Mean square error and bias of estimates based on 200 replications of the two-component mixtures of linear regression models when the true regression lines are parallel and the algorithms are initiated by random numbers (second strategy).

\begin{tabular}{|c|c|c|c|c|c|c|c|c|c|c|c|}
\hline$n$ & $\pi_{1}$ & Algorithm & & $\beta_{10}$ & $\beta_{11}$ & $\beta_{20}$ & $\beta_{21}$ & $\sigma_{1}^{2}$ & $\sigma_{2}^{2}$ & $\pi_{1}$ & $\pi_{2}$ \\
\hline \multirow[t]{18}{*}{50} & \multirow[t]{6}{*}{0.2} & \multirow[t]{2}{*}{ EM } & BIAS & 0.7827 & 0.0502 & 0.0211 & -0.0564 & 0.1880 & -0.1181 & 0.0969 & -0.0969 \\
\hline & & & MSE & 1.7402 & 0.3592 & 0.0987 & 0.1753 & 0.3768 & 0.1028 & 0.0341 & 0.0341 \\
\hline & & \multirow[t]{2}{*}{ CEM } & BIAS & 1.0966 & 0.4654 & 0.3985 & -0.5226 & 0.3458 & -0.2118 & 0.3477 & -0.3477 \\
\hline & & & MSE & 1.9298 & 1.0163 & 0.8319 & 0.8412 & 0.3372 & 0.3206 & 0.1752 & 0.1752 \\
\hline & & \multirow[t]{2}{*}{ SEM } & BIAS & 0.1730 & 0.0198 & 0.0513 & -0.0177 & -0.1871 & -0.1166 & 0.0552 & -0.0552 \\
\hline & & & MSE & 1.0832 & 0.1869 & 0.1342 & 0.0789 & 0.2186 & 0.0899 & 0.0392 & 0.0392 \\
\hline & \multirow[t]{6}{*}{0.5} & \multirow[t]{2}{*}{ EM } & BIAS & 0.2336 & 0.4011 & -0.3344 & -0.3640 & 0.0894 & 0.1185 & -0.0092 & 0.0092 \\
\hline & & & MSE & 0.5163 & 0.6505 & 0.6628 & 0.5733 & 0.2324 & 0.2502 & 0.0209 & 0.0209 \\
\hline & & \multirow[t]{2}{*}{ CEM } & BIAS & 0.5251 & 1.3023 & -0.5596 & -1.2973 & 0.2888 & 0.2471 & 0.0049 & -0.0049 \\
\hline & & & MSE & 0.6978 & 2.0004 & 0.7411 & 2.0337 & 0.2524 & 0.2068 & 0.0153 & 0.0153 \\
\hline & & \multirow[t]{2}{*}{ SEM } & BIAS & 0.0008 & 0.2155 & -0.1252 & -0.1599 & -0.0877 & -0.0340 & -0.0067 & 0.0067 \\
\hline & & & MSE & 0.3433 & 0.3820 & 0.4241 & 0.3291 & 0.1513 & 0.1477 & 0.0217 & 0.0217 \\
\hline & \multirow[t]{6}{*}{0.7} & \multirow[t]{2}{*}{ EM } & BIAS & 0.0512 & 0.1477 & -0.4307 & -0.1780 & -0.0323 & 0.0722 & -0.0550 & 0.0550 \\
\hline & & & MSE & 0.1646 & 0.2467 & 1.1040 & 0.4838 & 0.1184 & 0.2540 & 0.0245 & 0.0245 \\
\hline & & \multirow[t]{2}{*}{ CEM } & BIAS & 0.2785 & 1.1317 & -0.8648 & -1.2046 & 0.2091 & 0.1918 & -0.1735 & 0.1735 \\
\hline & & & MSE & 0.3948 & 1.8158 & 1.7031 & 1.8912 & 0.2281 & 0.2122 & 0.0629 & 0.0629 \\
\hline & & \multirow[t]{2}{*}{ SEM } & BIAS & 0.0314 & 0.0875 & -0.0536 & -0.1063 & -0.0494 & -0.1835 & -0.0161 & 0.0161 \\
\hline & & & MSE & 0.1868 & 0.1966 & 0.6563 & 0.2706 & 0.0906 & 0.1849 & 0.0236 & 0.0236 \\
\hline \multirow[t]{18}{*}{100} & \multirow[t]{6}{*}{0.2} & \multirow[t]{2}{*}{ EM } & BIAS & 0.4352 & 0.0330 & 0.0392 & -0.0255 & 0.1570 & -0.0574 & 0.0517 & -0.0517 \\
\hline & & & MSE & 0.9574 & 0.1198 & 0.0410 & 0.0304 & 0.3114 & 0.0309 & 140 & 0.0140 \\
\hline & & CEM & BIAS & 1.0709 & 0.4608 & 0.4155 & -0.4089 & 0.3381 & -0.2240 & 0.3580 & -0.3580 \\
\hline & & & MSE & 1.9736 & 0.7827 & 0.6706 & 0.6638 & 0.3967 & 0.3724 & 0.2076 & 0.2076 \\
\hline & & SEM & BIAS & -0.0012 & 0.0201 & 0.0226 & -0.0357 & -0.1848 & -0.0407 & 0.0228 & -0.0228 \\
\hline & & & MSE & 0.6221 & 0.1138 & 0.0628 & 0.0588 & 0.1404 & 0.0704 & 0.0259 & 0.0259 \\
\hline & 0.5 & EM & BIAS & 0.3370 & 0.4191 & -0.3635 & -0.3957 & 0.1559 & 0.1443 & 0.0052 & -0.0052 \\
\hline & & & MSE & 0.5714 & 0.6214 & 0.5851 & 0.6181 & 0.2524 & 0.2491 & 0.0270 & 0.0270 \\
\hline & & CEM & BIAS & 0.5293 & 1.0149 & -0.5920 & -1.0892 & 0.3023 & 0.3298 & -0.0002 & 0.0002 \\
\hline & & & MSE & 0.6020 & 1.9793 & 0.6950 & 1.9316 & 0.2379 & 0.2497 & 0.0183 & 0.0183 \\
\hline & & SEM & BIAS & 0.0368 & 0.2438 & -0.0910 & -0.2023 & -0.0499 & -0.0114 & -0.0108 & 0.0108 \\
\hline & & & MSE & 0.1656 & 0.3893 & 0.2061 & 0.3425 & 0.0707 & 0.0910 & 0.0116 & 0.0116 \\
\hline & 0.7 & EM & BIAS & 0.0251 & 0.1250 & -0.2805 & -0.1037 & & 0.0659 & -0.0 & 0.0400 \\
\hline & & & MSE & 0.0777 & 0.1667 & 0.5957 & 0.1985 & 0.0655 & 0.1469 & 0.0160 & 0.0160 \\
\hline & & CEM & BIAS & 0.3008 & 1.1962 & -0.9133 & -1.1590 & 0.2646 & 0.2119 & -0.1720 & 0.1720 \\
\hline & & & MSE & 0.2575 & 1.8363 & 1.4507 & 1.7035 & 0.2088 & 0.2241 & 0.0598 & 0.0598 \\
\hline & & SEM & BIAS & 0.0201 & 0.1477 & -0.0581 & -0.1242 & -0.0156 & -0.1037 & -0.0193 & 0.0193 \\
\hline & & & MSE & 0.0901 & 0.2322 & 0.3495 & 0.2564 & 0.0518 & 0.0818 & 0.0144 & 0.0144 \\
\hline 500 & 0.2 & EM & & & & & 10 & & 33 & & 086 \\
\hline & & & MSE & 0.1236 & 0.0124 & 0.0061 & 0.0021 & 0.0496 & 0.0027 & 0.0013 & 0.0013 \\
\hline & & CEM & BIAS & 0.4715 & 0.1545 & 0.4745 & -0.0809 & 0.3007 & -0.3661 & 0.3711 & -0.3711 \\
\hline & & & MSE & 1.2340 & 0.2662 & 0.8383 & 0.1586 & 0.4994 & 0.5526 & 0.2969 & 0.2969 \\
\hline & & SEM & BIAS & -0.1490 & 0.0022 & 0.0103 & -0.0002 & -0.1452 & -0.0188 & -0.0061 & 0.0061 \\
\hline & & & MSE & 0.0943 & 0.0294 & 0.0093 & 0.0020 & 0.0304 & 0.0060 & 0.0006 & 0.0006 \\
\hline & 0.5 & EM & BIAS & 0.4643 & 0.3850 & -0.4349 & -0.4198 & 0.2545 & 0.2477 & 0.0061 & -0.0061 \\
\hline & & & MSE & 0.7181 & 0.5459 & 0.6619 & 0.6290 & 0.3586 & 0.3133 & 0.0455 & 0.0455 \\
\hline & & CEM & BIAS & 0.5275 & 1.4251 & -0.5480 & -1.4142 & 0.2880 & 0.3503 & -0.0040 & 0.0040 \\
\hline & & & MSE & 0.4227 & 2.1883 & 0.4455 & 2.1539 & 0.2416 & 0.2822 & 0.0254 & 0.0254 \\
\hline & & SEM & BIAS & 0.0920 & 0.2721 & -0.0645 & -0.3022 & 0.0251 & 0.0021 & 0.0068 & -0.0068 \\
\hline & & & MSE & 0.1759 & 0.4298 & 0.0942 & 0.4779 & 0.0849 & 0.0852 & 0.0111 & 0.0111 \\
\hline & 0.7 & EM & BIAS & 0.0078 & 0.0100 & -0.0194 & -0.0041 & -0.0002 & -0.0059 & -0.0021 & 0.0021 \\
\hline & & & MSE & 0.0086 & 0.0177 & 0.0480 & 0.0237 & 0.0073 & 0.0106 & 0.0018 & 0.0018 \\
\hline & & CEM & BIAS & 0.3464 & 0.8679 & -0.5439 & -0.7969 & 0.2809 & 0.0540 & -0.0964 & 0.0964 \\
\hline & & & MSE & 0.2765 & 1.3092 & 1.2023 & 1.2654 & 0.3002 & 0.2831 & 0.0630 & 0.0630 \\
\hline & & SEM & BIAS & 0.2992 & 0.4658 & 0.6887 & 0.4997 & 0.3220 & 0.3316 & 0.1677 & 0.1677 \\
\hline & & & MSE & 0.0946 & 0.2365 & 0.4792 & 0.2651 & 0.1032 & 0.1153 & 0.0284 & 0.0284 \\
\hline
\end{tabular}


Table 6. Mean square error and bias of estimates based on 200 replications of the two-component mixtures of linear regression models when the true regression lines are concurrent and the true values are used as the starting values.

\begin{tabular}{|c|c|c|c|c|c|c|c|c|c|c|c|}
\hline$n$ & $\pi_{1}$ & Algorithm & & $\beta_{10}$ & $\beta_{11}$ & $\beta_{20}$ & $\beta_{21}$ & $\sigma_{1}^{2}$ & $\sigma_{2}^{2}$ & $\pi_{1}$ & $\pi_{2}$ \\
\hline \multirow[t]{18}{*}{50} & \multirow[t]{6}{*}{0.2} & \multirow[t]{2}{*}{ EM } & BIAS & -0.0018 & 0.0052 & -0.0032 & -0.0029 & -0.0343 & -0.0065 & 0.0022 & -0.0022 \\
\hline & & & MSE & 0.0123 & 0.0065 & 0.0021 & 0.0007 & 0.0043 & 0.0006 & 0.0040 & 0.0040 \\
\hline & & \multirow[t]{2}{*}{ CEM } & BIAS & 0.0051 & -0.0105 & -0.0041 & -0.0023 & -0.0380 & -0.0087 & -0.0160 & 0.0160 \\
\hline & & & MSE & 0.0115 & 0.0066 & 0.0021 & 0.0007 & 0.0044 & 0.0006 & 0.0040 & 0.0040 \\
\hline & & \multirow[t]{2}{*}{ SEM } & BIAS & 0.0024 & -0.0095 & -0.0044 & -0.0020 & -0.0389 & -0.0079 & 0.0015 & -0.0015 \\
\hline & & & MSE & 0.0122 & 0.0066 & 0.0020 & 0.0007 & 0.0046 & 0.0006 & 0.0045 & 0.0045 \\
\hline & \multirow[t]{6}{*}{0.5} & \multirow[t]{2}{*}{ EM } & BIAS & -0.0061 & -0.0029 & 0.0064 & -0.0040 & -0.0077 & -0.0108 & 0.0081 & -0.0081 \\
\hline & & & MSE & 0.0032 & 0.0012 & 0.0037 & 0.0015 & 0.0011 & 0.0011 & 0.0054 & 0.0054 \\
\hline & & \multirow[t]{2}{*}{ CEM } & BIAS & -0.0044 & -0.0050 & 0.0029 & -0.0015 & -0.0129 & -0.0160 & 0.0097 & -0.0097 \\
\hline & & & MSE & 0.0033 & 0.0013 & 0.0035 & 0.0014 & 0.0012 & 0.0012 & 0.0075 & 0.0075 \\
\hline & & \multirow[t]{2}{*}{ SEM } & BIAS & -0.0044 & -0.0051 & 0.0026 & -0.0014 & -0.0114 & -0.0150 & 0.0071 & -0.0071 \\
\hline & & & MSE & 0.0033 & 0.0013 & 0.0038 & 0.0014 & 0.0012 & 0.0013 & 0.0063 & 0.0063 \\
\hline & \multirow[t]{6}{*}{0.7} & \multirow[t]{2}{*}{ EM } & BIAS & -0.0033 & 0.0025 & 0.0059 & -0.0055 & -0.0060 & -0.0186 & -0.0053 & 0.0053 \\
\hline & & & MSE & 0.0024 & 0.0008 & 0.0065 & 0.0026 & 0.0007 & 0.0022 & 0.0046 & 0.0046 \\
\hline & & \multirow[t]{2}{*}{ CEM } & BIAS & -0.0027 & 0.0015 & 0.0024 & -0.0028 & -0.0101 & -0.0221 & 0.0145 & -0.0145 \\
\hline & & & MSE & 0.0023 & 0.0008 & 0.0068 & 0.0026 & 0.0007 & 0.0023 & 0.0049 & 0.0049 \\
\hline & & \multirow[t]{2}{*}{ SEM } & BIAS & -0.0025 & 0.0014 & 0.0008 & -0.0019 & -0.0083 & -0.0229 & -0.0024 & 0.0024 \\
\hline & & & MSE & 0.0024 & 0.0008 & 0.0073 & 0.0026 & 0.0008 & 0.0023 & 0.0051 & 0.0051 \\
\hline \multirow[t]{18}{*}{100} & \multirow[t]{6}{*}{0.2} & \multirow[t]{2}{*}{ EM } & BIAS & -0.0025 & 0.0024 & 0.0026 & -0.0011 & -0.0186 & -0.0044 & -0.0 & 0.0033 \\
\hline & & & MSE & 0.0049 & 0.0020 & 0.0011 & 0.0005 & 0.0017 & 0.0003 & 0.0017 & 0.0017 \\
\hline & & CEM & BIAS & 0.0004 & -0.0006 & 0.0018 & -0.0006 & -0.0228 & -0.0067 & -0.0237 & 0.0237 \\
\hline & & & MSE & 0.0050 & 0.0020 & 0.0010 & 0.0005 & 0.0018 & 0.0003 & 0.0021 & 0.0021 \\
\hline & & SEM & BIAS & 0.0019 & -0.0008 & 0.0018 & -0.0002 & -0.0234 & -0.0067 & -0.0049 & 0.0049 \\
\hline & & & MSE & 0.0049 & 0.0020 & 0.0010 & 0.0005 & 0.0019 & 0.0004 & 0.0018 & 0.0018 \\
\hline & 0.5 & EM & BIAS & 0.0026 & 0.0008 & 0.0009 & -0.0006 & -0.0104 & -0.0061 & 0.0046 & -0.0046 \\
\hline & & & MSE & 0.0016 & 0.0006 & 0.0017 & 0.0006 & 0.0006 & 0.0007 & 0.0031 & 0.0031 \\
\hline & & CEM & BIAS & 0.0044 & -0.0011 & -0.0009 & 0.0015 & -0.0155 & -0.0114 & 0.0049 & -0.0049 \\
\hline & & & MSE & 0.0016 & 0.0006 & 0.0017 & 0.0006 & 0.0007 & 0.0008 & 45 & 0.0045 \\
\hline & & SEM & BIAS & 0.0042 & -0.0010 & -0.0007 & 0.0015 & -0.0139 & -0.0098 & 0.0063 & -0.0063 \\
\hline & & & MSE & 0.0017 & 0.0006 & 0.0017 & 0.0006 & 0.0007 & 0.0007 & 0.0035 & 0.0035 \\
\hline & 0.7 & EM & BIAS & -0.0030 & -0.0008 & -0.0038 & 0.0006 & -0.0029 & -0.0080 & -0.0013 & 0.0013 \\
\hline & & & MSE & 0.0012 & 0.0005 & 0.0030 & 0.0010 & 0.0003 & 0.0009 & 0.0024 & 0.0024 \\
\hline & & CEM & BIAS & -0.0021 & -0.0018 & -0.0060 & 0.0034 & -0.0070 & -0.0120 & 0.0204 & -0.0204 \\
\hline & & & MSE & 0.0012 & 0.0005 & 0.0030 & 0.0010 & 0.0004 & 0.0010 & 0.0029 & 0.0029 \\
\hline & & SEM & BIAS & -0.0018 & -0.0021 & -0.0047 & 0.0028 & -0.0059 & -0.0124 & -0.0025 & 0.0025 \\
\hline & & & MSE & 0.0012 & 0.0005 & 0.0030 & 0.0010 & 0.0004 & 0.0010 & 0.0027 & 0.0027 \\
\hline 500 & 0.2 & EM & BIAS & -0.0044 & 0.0017 & -0.0005 & 0.0000 & -0.0022 & -0.0013 & -0.0 & \\
\hline & & & MSE & 0.0010 & 0.0003 & 0.0002 & 0.0001 & 0.0003 & 0.0000 & 0.0003 & 0.0003 \\
\hline & & CEM & BIAS & -0.0004 & -0.0015 & -0.0013 & 0.0007 & -0.0053 & -0.0043 & -0.0211 & 0.0211 \\
\hline & & & MSE & 0.0010 & 0.0003 & 0.0002 & 0.0001 & 0.0003 & 0.0001 & 0.0008 & 0.0008 \\
\hline & & SEM & BIAS & -0.0009 & -0.0013 & -0.0018 & 0.0012 & -0.0057 & -0.0043 & -0.0006 & 0.0006 \\
\hline & & & MSE & 0.0010 & 0.0003 & 0.0002 & 0.0001 & 0.0003 & 0.0001 & 0.0004 & 0.0004 \\
\hline & 0.5 & EM & BIAS & 0.0017 & -0.0004 & 0.0016 & -0.0011 & -0.0012 & -0.0003 & 0.0013 & -0.0013 \\
\hline & & & MSE & 0.0003 & 0.0001 & 0.0003 & 0.0001 & 0.0001 & 0.0001 & 0.0006 & 0.0006 \\
\hline & & CEM & BIAS & 0.0038 & -0.0024 & -0.0009 & 0.0011 & -0.0066 & -0.0059 & 0.0024 & -0.0024 \\
\hline & & & MSE & 0.0003 & 0.0001 & 0.0003 & 0.0001 & 0.0001 & 0.0001 & 0.0012 & 0.0012 \\
\hline & & SEM & BIAS & 0.0035 & -0.0024 & -0.0005 & 0.0009 & -0.0050 & -0.0041 & 0.0010 & -0.0010 \\
\hline & & & MSE & 0.0003 & 0.0001 & 0.0003 & 0.0001 & 0.0001 & 0.0001 & 0.0007 & 0.0007 \\
\hline & 0.7 & EM & BIAS & 0.0012 & -0.0004 & -0.0015 & 0.0007 & -0.0013 & -0.0032 & 0.0009 & -0.0009 \\
\hline & & & MSE & 0.0003 & 0.0001 & 0.0006 & 0.0002 & 0.0001 & 0.0002 & 0.0005 & 0.0005 \\
\hline & & CEM & BIAS & 0.0024 & -0.0015 & -0.0046 & 0.0035 & -0.0058 & -0.0068 & 0.0243 & -0.0243 \\
\hline & & & MSE & 0.0003 & 0.0001 & 0.0006 & 0.0002 & 0.0001 & 0.0002 & 0.0011 & 0.0011 \\
\hline & & SEM & BIAS & 0.0028 & -0.0019 & -0.0047 & 0.0034 & -0.0048 & -0.0071 & -0.0005 & 0.0005 \\
\hline & & & MSE & 0.0003 & 0.0001 & 0.0006 & 0.0002 & 0.0001 & 0.0002 & 0.0005 & 0.0005 \\
\hline
\end{tabular}


Table 7. Mean square error and bias of estimates based on 200 replications of the two-component mixtures of linear regression models when the true regression lines are concurrent and the algorithms are initiated by random numbers (second strategy).

\begin{tabular}{|c|c|c|c|c|c|c|c|c|c|c|c|}
\hline$n$ & $\pi_{1}$ & Algorithm & & $\beta_{10}$ & $\beta_{11}$ & $\beta_{20}$ & $\beta_{21}$ & $\sigma_{1}^{2}$ & $\sigma_{2}^{2}$ & $\pi_{1}$ & $\pi_{2}$ \\
\hline \multirow[t]{18}{*}{50} & \multirow[t]{6}{*}{0.2} & \multirow[t]{2}{*}{ EM } & BIAS & -0.0069 & 0.0112 & -0.0037 & -0.0025 & -0.0196 & -0.0078 & 0.0040 & -0.0040 \\
\hline & & & MSE & 0.0176 & 0.0121 & 0.0021 & 0.0007 & 0.0099 & 0.0006 & 0.0039 & 0.0039 \\
\hline & & \multirow[t]{2}{*}{ CEM } & BIAS & -0.6851 & 0.5164 & 0.4327 & -0.2542 & 0.0923 & 0.1185 & 0.1246 & -0.1246 \\
\hline & & & MSE & 0.9278 & 0.4210 & 0.4363 & 0.1193 & 0.0379 & 0.0315 & 0.0509 & 0.0509 \\
\hline & & \multirow[t]{2}{*}{ SEM } & BIAS & -0.0288 & 0.0383 & -0.0003 & -0.0057 & -0.0157 & -0.0083 & 0.0101 & -0.0101 \\
\hline & & & MSE & 0.0471 & 0.0438 & 0.0075 & 0.0025 & 0.0131 & 0.0022 & 0.0081 & 0.0081 \\
\hline & \multirow[t]{6}{*}{0.5} & \multirow[t]{2}{*}{ EM } & BIAS & -0.0061 & -0.0029 & 0.0064 & -0.0040 & -0.0077 & -0.0108 & 0.0081 & -0.0081 \\
\hline & & & MSE & 0.0032 & 0.0012 & 0.0037 & 0.0015 & 0.0011 & 0.0011 & 0.0054 & 0.0054 \\
\hline & & \multirow[t]{2}{*}{ CEM } & BIAS & -1.0466 & 0.5469 & 1.0231 & -0.5635 & 0.1973 & 0.2014 & -0.0012 & 0.0012 \\
\hline & & & MSE & 1.2271 & 0.3168 & 1.1964 & 0.3374 & 0.0512 & 0.0540 & 0.0141 & 0.0141 \\
\hline & & \multirow[t]{2}{*}{ SEM } & BIAS & -0.0761 & 0.0334 & 0.0955 & -0.0502 & 0.0088 & -0.0002 & 0.0092 & -0.0092 \\
\hline & & & MSE & 0.0770 & 0.0213 & 0.1229 & 0.0328 & 0.0087 & 0.0052 & 0.0083 & 0.0083 \\
\hline & \multirow[t]{6}{*}{0.7} & \multirow[t]{2}{*}{ EM } & BIAS & -0.0059 & 0.0053 & 0.0127 & -0.0142 & -0.0024 & -0.0162 & -0.0051 & 0.0051 \\
\hline & & & MSE & 0.0031 & 0.0017 & 0.0202 & 0.0088 & 0.0026 & 0.0024 & 0.0047 & 0.0047 \\
\hline & & \multirow[t]{2}{*}{ CEM } & BIAS & -0.8668 & 0.4435 & 0.9672 & -0.6610 & 0.1689 & 0.1953 & -0.1613 & 0.1613 \\
\hline & & & MSE & 0.9454 & 0.2238 & 1.2199 & 0.4807 & 0.0455 & 0.0640 & 0.0541 & 0.0541 \\
\hline & & \multirow[t]{2}{*}{ SEM } & BIAS & -0.0166 & 0.0144 & 0.0173 & -0.0220 & 0.0012 & -0.0205 & -0.0027 & 0.0027 \\
\hline & & & MSE & 0.0158 & 0.0074 & 0.0341 & 0.0172 & 0.0046 & 0.0029 & 0.0059 & 0.0059 \\
\hline \multirow[t]{18}{*}{100} & \multirow[t]{6}{*}{0.2} & \multirow[t]{2}{*}{ EM } & BIAS & -0.0060 & 0.0051 & 0.0027 & -0.0011 & -0.0156 & -0.0046 & -0.0030 & 0.0030 \\
\hline & & & MSE & 0.0076 & 0.0 & 0.0011 & 0.0 & 0.0 & 0.0 & 0.0 & 0.0017 \\
\hline & & CEM & BIAS & -0.5196 & 0.3564 & 0.4082 & -0.2055 & 0.0599 & 0.1138 & 0.0716 & -0.0716 \\
\hline & & & MSE & 0.6721 & 0.2791 & 0.4327 & 0.0984 & 0.0252 & 0.0296 & 0.0378 & 0.0378 \\
\hline & & SEM & BIAS & -0.0099 & 0.0068 & 0.0028 & -0.0008 & -0.0170 & -0.0060 & -0.0002 & 0.0002 \\
\hline & & & MSE & 0.0132 & 0.0053 & 0.0015 & 0.0006 & 0.0056 & 0.0008 & 0.0022 & 0.0022 \\
\hline & 0.5 & EM & BIAS & 0.0026 & 0.0008 & 0.0009 & -0.0006 & -0.0104 & -0.0061 & 0.0046 & -0.0046 \\
\hline & & & MSE & 0.0016 & & & & & & & 0.0031 \\
\hline & & CEM & BIAS & -1.1072 & 0.5752 & 1.1117 & -0.5815 & 0.2173 & 0.2147 & -0.0010 & 0.0010 \\
\hline & & & MSE & 1.2838 & 0.3363 & 1.2994 & 0.3439 & 0.0561 & 0.0546 & 0.0113 & 0.0113 \\
\hline & & SEM & BIAS & -0.1006 & 0.0576 & 0.1272 & -0.0593 & 0.0133 & 0.0087 & 0.0083 & -0.0083 \\
\hline & & & MSE & 0.1163 & 0.0355 & 0.1667 & 0.0377 & 0.0082 & 0.0052 & 0.0048 & 0.0048 \\
\hline & 0.7 & EM & BIAS & -0.0034 & -0.0007 & -0.0019 & -0.0004 & -0.0033 & -0.0059 & -0.0015 & 0.0015 \\
\hline & & & MSE & 0.0012 & 0.0005 & 0.0039 & 0.0012 & 0.0003 & 0.0018 & 24 & 0.0024 \\
\hline & & CEM & BIAS & -0.8463 & 0.4298 & 0.9218 & -0.6125 & 0.1772 & 0.1858 & -0.1346 & 0.1346 \\
\hline & & & MSE & 0.9533 & 0.2210 & 1.1923 & 0.4331 & 0.0485 & 0.0595 & 0.0477 & 0.0477 \\
\hline & & SEM & BIAS & -0.0354 & 0.0156 & 0.0410 & -0.0215 & 0.0019 & -0.0038 & -0.0022 & 0.0022 \\
\hline & & & MSE & 0.0314 & 0.0092 & 0.0647 & 0.0174 & 0.0025 & 0.0030 & 0.0031 & 0.0031 \\
\hline 500 & 0.2 & EM & BIAS & -0.0 & & 0. & & $-0 .($ & -0 & -0 & 0.0008 \\
\hline & & & MSE & 0.0010 & 0.0003 & 002 & 0.0001 & 03 & 0.0000 & 03 & 0.0003 \\
\hline & & CEM & BIAS & -0.0269 & 0.0337 & 0.0376 & -0.0191 & 0.0028 & 0.0076 & -0.0127 & 0.0127 \\
\hline & & & MSE & 0.0432 & 0.0269 & 0.0453 & 0.0093 & 0.0030 & 0.0037 & 0.0054 & 0.0054 \\
\hline & & SEM & BIAS & -0.0073 & 0.0024 & 0.0035 & -0.0013 & -0.0044 & -0.0033 & 0.0003 & -0.0003 \\
\hline & & & MSE & 0.0087 & 0.0027 & 0.0053 & 0.0012 & 0.0005 & 0.0003 & 0.0008 & 0.0008 \\
\hline & 0.5 & EM & BIAS & 0.0017 & -0.0004 & 0.0016 & -0.0011 & -0.0012 & -0.0003 & 0.0013 & -0.0013 \\
\hline & & & MSE & 0.0003 & 0.0001 & 0.0003 & 0.0001 & 0.0001 & 0.0001 & 0.0006 & 0.0006 \\
\hline & & CEM & BIAS & -1.1192 & 0.5721 & 1.1164 & -0.5785 & 0.2186 & 0.2319 & -0.0061 & 0.0061 \\
\hline & & & MSE & 1.3032 & 0.3310 & 1.2951 & 0.3366 & 0.0557 & 0.0624 & 0.0112 & 0.0112 \\
\hline & & SEM & BIAS & -0.0133 & 0.0070 & 0.0179 & -0.0081 & -0.0013 & -0.0009 & & -0.0021 \\
\hline & & & MSE & 0.0191 & 0.0058 & 0.0239 & 0.0058 & 0.0010 & 0.0007 & 0.0007 & 0.0007 \\
\hline & 0.7 & EM & BIAS & 0.0012 & -0.0004 & -0.0015 & 0.0007 & -0.0013 & -0.0032 & 0.0009 & -0.0009 \\
\hline & & & MSE & 0.0003 & 0.0001 & 0.0006 & 0.0002 & 0.0001 & 0.0002 & 0.0005 & 0.0005 \\
\hline & & CEM & BIAS & -0.4957 & 0.2525 & 0.6172 & -0.3798 & 0.1307 & 0.1050 & -0.0524 & 0.0524 \\
\hline & & & MSE & 0.5283 & 0.1217 & 0.8541 & 0.2704 & 0.0448 & 0.0372 & 0.0332 & 0.0332 \\
\hline & & SEM & BIAS & -0.0054 & 0.0029 & 0.0160 & -0.0057 & 0.0002 & -0.0056 & 0.0024 & -0.0024 \\
\hline & & & MSE & 0.0049 & 0.0017 & 0.0291 & 0.0057 & 0.0017 & 0.0004 & 0.0006 & 0.0006 \\
\hline
\end{tabular}



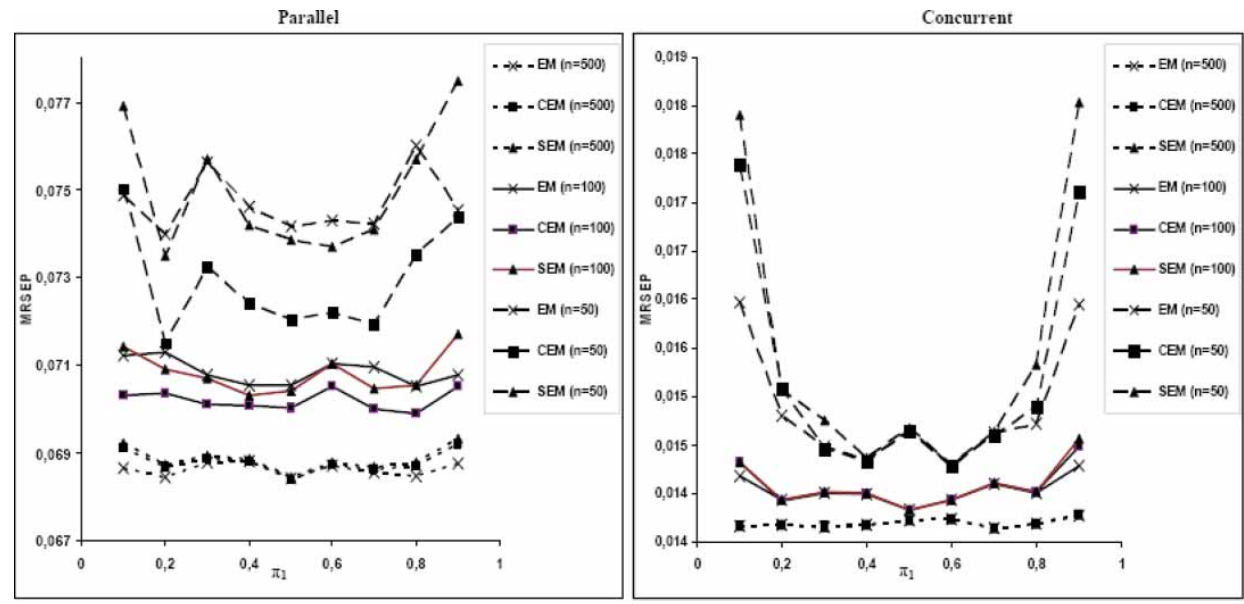

Figure 2. MRSEP by 10-fold cross-validation for two-component models when the true values were used as the starting values.

Parallel

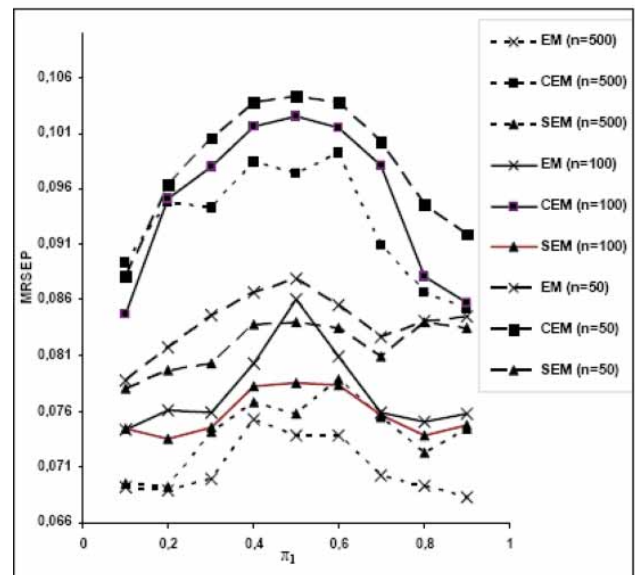

Concurrent

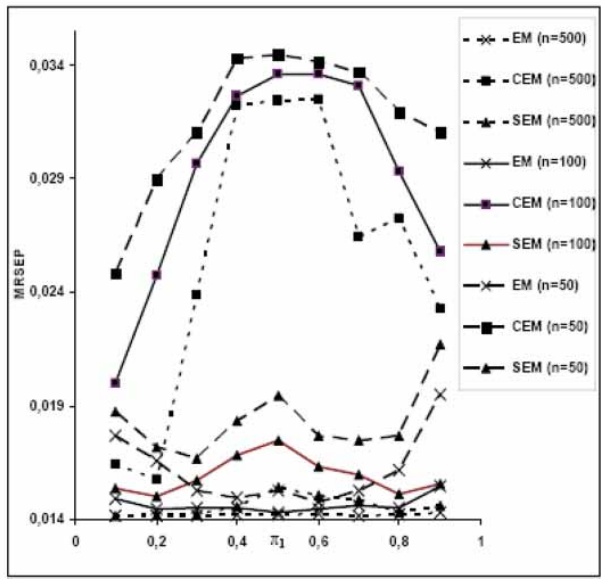

Figure 3. MRSEP by 10-fold cross-validation for two-component models when the algorithms were initiated by random numbers (second strategy).

Table 8. True parameter values for the essays with a three-component mixture of linear regressions.

\begin{tabular}{lccccccccc}
\hline Configuration & $\beta_{01}$ & $\beta_{02}$ & $\beta_{03}$ & $\beta_{11}$ & $\beta_{12}$ & $\beta_{13}$ & $\sigma_{1}^{2}$ & $\sigma_{2}^{2}$ & $\sigma_{3}^{2}$ \\
\hline Parallel & -1 & 1 & 0 & 1 & 1 & 1 & $0.2^{2}$ & $0.2^{2}$ & $0.2^{2}$ \\
Concurrent & -1 & 3 & 3 & 1 & -1 & 1 & $0.5^{2}$ & $1^{2}$ & $0.3^{2}$ \\
\hline
\end{tabular}

Tables 9 and 10 report the mean number of iterations required for convergence using the EM and CEM algorithms for fitting three-component mixtures of linear regression models. Also in all cases, the mean number of iterations for convergence is smaller using the CEM algorithm rather than using the EM algorithm. 


\section{Parallel}

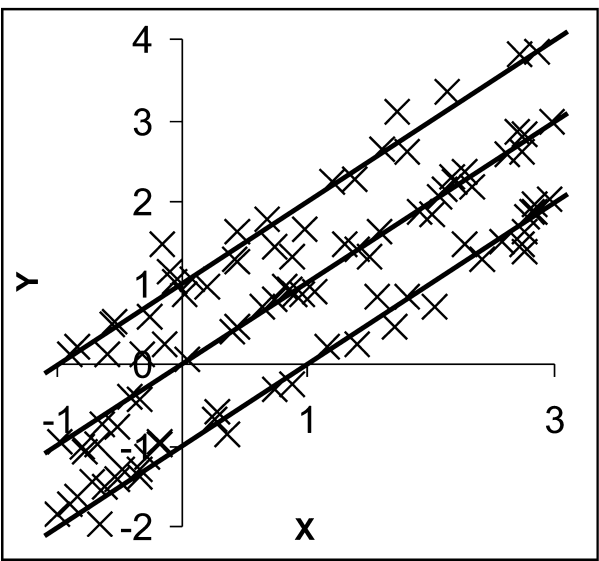

Concurrent

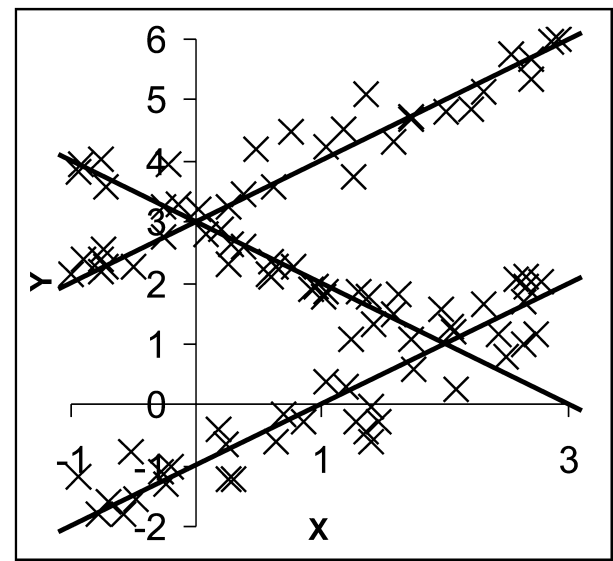

Figure 4. Scatter plot of samples from three-component models with $n=100$ and $\pi=(0.4 ; 0.3 ; 0.3)$.

Table 9. The mean number of iterations required for convergence using the EM and CEM algorithms for three-component mixtures of linear regressions when the true values were used as the starting values.

\begin{tabular}{|c|c|c|c|c|c|c|c|c|}
\hline \multirow[b]{3}{*}{$\left(\pi_{1} ; \pi_{2}\right)$} & \multicolumn{4}{|c|}{ Parallel } & \multicolumn{4}{|c|}{ Concurrent } \\
\hline & \multicolumn{2}{|c|}{$n=100$} & \multicolumn{2}{|c|}{$n=500$} & \multicolumn{2}{|c|}{$n=100$} & \multicolumn{2}{|c|}{$n=500$} \\
\hline & EM & CEM & EM & CEM & EM & CEM & EM & CEM \\
\hline$(0.2 ; 0.2)$ & 20.71 & 10.55 & 12.26 & 10.19 & 26.18 & 12.99 & 17.04 & 12.79 \\
\hline$(0.2 ; 0.3)$ & 18.79 & 9.87 & 11.79 & 9.65 & 25.07 & 13.30 & 15.71 & 13.40 \\
\hline$(0.2 ; 0.4)$ & 16.41 & 9.57 & 11.93 & 10.24 & 23.95 & 13.99 & 17.30 & 15.36 \\
\hline$(0.2 ; 0.5)$ & 19.31 & 10.12 & 12.09 & 10.26 & 27.39 & 15.21 & 17.61 & 16.02 \\
\hline$(0.2 ; 0.6)$ & 19.71 & 9.86 & 12.94 & 10.73 & 31.76 & 14.91 & 18.81 & 16.40 \\
\hline$(0.3 ; 0.2)$ & 18.41 & 10.21 & 12.39 & 9.64 & 24.43 & 12.92 & 15.87 & 12.59 \\
\hline$(0.3 ; 0.3)$ & 19.75 & 10.33 & 12.12 & 10.09 & 22.47 & 13.64 & 15.61 & 13.09 \\
\hline$(0.3 ; 0.4)$ & 17.79 & 9.62 & 12.33 & 10.59 & 25.84 & 13.86 & 15.22 & 14.18 \\
\hline$(0.3 ; 0.5)$ & 18.60 & 9.62 & 13.60 & 11.12 & 27.31 & 14.58 & 16.53 & 15.04 \\
\hline$(0.4 ; 0.2)$ & 17.61 & 9.96 & 12.13 & 10.12 & 21.11 & 12.38 & 16.26 & 12.92 \\
\hline$(0.4 ; 0.3)$ & 19.38 & 9.99 & 12.82 & 10.71 & 20.95 & 13.28 & 15.63 & 13.50 \\
\hline$(0.4 ; 0.4)$ & 20.70 & 9.61 & 13.15 & 10.84 & 23.36 & 14.22 & 15.70 & 13.88 \\
\hline$(0.5 ; 0.2)$ & 17.32 & 9.93 & 12.57 & 10.43 & 24.09 & 13.37 & 16.95 & 12.94 \\
\hline$(0.5 ; 0.3)$ & 18.87 & 9.47 & 13.63 & 11.00 & 22.89 & 13.34 & 15.44 & 13.46 \\
\hline$(0.6 ; 0.2)$ & 21.85 & 9.90 & 13.00 & 10.66 & 24.29 & 13.64 & 16.55 & 12.49 \\
\hline
\end{tabular}

Tables 11-14 provide the MSE and the bias of the parameter estimates over the 200 replications of the three-component mixtures of linear regression models, when the mixing proportion $\left(\pi_{1} ; \pi_{2} ; \pi_{3}\right)$ is chosen to be $(0.2 ; 0.2 ; 0.6),(0.2 ; 0.4 ; 0.4),(0.3 ; 0.3 ; 0.4)$ and $(0.5 ; 0.3 ; 0.2)$.

When the true regression lines are parallel and the algorithms are initiated with the true parameter values, Table 11 shows that the CEM parameter estimates have smaller MSE than the EM and SEM estimates and EM and SEM have practically the same behaviour. However, for samples of size 500, the estimates MSE are identical. Also, as in the previous cases, the MSE decreases when the sample size increases. 
Table 10. The mean number of iterations required for convergence using the EM and CEM algorithms for three-component mixtures of linear regressions when the algorithms were initiated by random numbers (second strategy).

\begin{tabular}{|c|c|c|c|c|c|c|c|c|}
\hline \multirow[b]{3}{*}{$\left(\pi_{1} ; \pi_{2}\right)$} & \multicolumn{4}{|c|}{ Parallel } & \multicolumn{4}{|c|}{ Concurrent } \\
\hline & \multicolumn{2}{|c|}{$n=100$} & \multicolumn{2}{|c|}{$n=500$} & \multicolumn{2}{|c|}{$n=100$} & \multicolumn{2}{|c|}{$n=500$} \\
\hline & EM & CEM & EM & CEM & EM & CEM & EM & CEM \\
\hline$(0.2 ; 0.2)$ & 198.82 & 24.46 & 350.25 & 27.19 & 111.27 & 18.92 & 169.13 & 21.71 \\
\hline$(0.2 ; 0.3)$ & 177.28 & 24.98 & 266.03 & 28.05 & 88.50 & 20.96 & 152.50 & 24.76 \\
\hline$(0.2 ; 0.4)$ & 140.03 & 26.18 & 240.82 & 28.35 & 121.84 & 21.90 & 141.20 & 26.90 \\
\hline$(0.2 ; 0.5)$ & 137.92 & 25.21 & 250.67 & 29.67 & 227.42 & 22.53 & 258.78 & 27.82 \\
\hline$(0.2 ; 0.6)$ & 144.62 & 25.67 & 249.45 & 31.35 & 262.89 & 22.75 & 276.89 & 29.87 \\
\hline$(0.3 ; 0.2)$ & 190.24 & 26.73 & 260.08 & 29.89 & 77.97 & 18.80 & 115.63 & 23.45 \\
\hline$(0.3 ; 0.3)$ & 175.81 & 26.89 & 230.71 & 28.20 & 155.93 & 18.47 & 293.18 & 24.68 \\
\hline$(0.3 ; 0.4)$ & 141.42 & 25.97 & 243.78 & 30.56 & 213.99 & 21.99 & 297.67 & 23.37 \\
\hline$(0.3 ; 0.5)$ & 99.73 & 25.51 & 200.32 & 26.87 & 235.92 & 21.08 & 276.78 & 28.67 \\
\hline$(0.4 ; 0.2)$ & 137.32 & 26.57 & 225.48 & 28.51 & 175.65 & 19.29 & 197.56 & 27.65 \\
\hline$(0.4 ; 0.3)$ & 128.94 & 28.01 & 199.91 & 32.39 & 177.96 & 20.51 & 210.61 & 27.81 \\
\hline$(0.4 ; 0.4)$ & 112.19 & 28.74 & 198.67 & 31.33 & 152.96 & 22.76 & 206.53 & 28.25 \\
\hline$(0.5 ; 0.2)$ & 129.28 & 25.84 & 187.54 & 29.89 & 112.70 & 20.57 & 205.34 & 27.94 \\
\hline$(0.5 ; 0.3)$ & 97.90 & 27.75 & 155.65 & 36.88 & 91.59 & 19.82 & 68.49 & 26.53 \\
\hline$(0.6 ; 0.2)$ & 137.03 & 25.48 & 233.89 & 31.67 & 97.95 & 21.49 & 75.34 & 25.89 \\
\hline
\end{tabular}

When the true regression lines are parallel and the algorithms are initiated by random numbers (second strategy), Table 12 shows that the SEM algorithm performs better than the other two. Using this strategy as the starting value, the performance of all algorithms decreases by producing estimates of the parameters that have higher MSE and bias.

When the true regression lines are concurrent and the algorithms are initiated with the true parameter values, Table 13 shows that EM outperforms CEM and SEM by producing estimates of the parameters that have lower bias and smaller MSE. It seems that the MSE of CEM estimates of the regression coefficients and the variances are smaller than the MSE of SEM estimates. The MSE of the parameter estimates tends to approach zero as sample size increases.

When the true regression lines are concurrent but the algorithms are initiated by random numbers (second strategy), Table 14 shows that CEM estimates of the parameters have higher MSE than EM and SEM estimates. In generality, SEM outperforms EM by producing estimates of the parameters that have smaller MSE.

The resulting values of MRSEP based on 10-fold cross-validation when the true values were used as the starting value for each of the configurations of the true regression lines are plotted in Figure 5. Similar results were obtained calculating MRSEP based on 5-fold cross-validation. When the true regression lines are parallel, the CEM algorithm performs better, although in samples of size 500, the three algorithms have practically the same behaviour. Figure 5 suggests that the CEM algorithm performs always better in fitting a three-component mixture of linear regressions when the true regression lines are concurrent and the algorithms are initiated with the true parameter values.

The resulting values of MRSEP based on 10-fold cross-validation when the second strategy was used as the starting values for each of the configurations of the true regression lines are plotted in Figure 6. Similar results were obtained calculating MRSEP based on 5-fold cross-validation. In both configurations of the true regression lines, Figure 6 suggests that the SEM algorithm performs always better in fitting a three-component mixture of linear regressions. 
Table 11. Mean square error and bias of estimates based on 200 replications of the three-component mixtures of linear regression models when the true regression lines are parallel and the true values are used as the starting values.

\begin{tabular}{|c|c|c|c|c|c|c|c|c|c|c|c|c|c|c|c|}
\hline$n$ & $\left(\pi_{1} ; \pi_{2}\right)$ & Algorithm & & $\beta_{10}$ & $\beta_{11}$ & $\beta_{20}$ & $\beta_{21}$ & $\beta_{30}$ & $\beta_{31}$ & $\sigma_{1}$ & $\sigma_{2}$ & $\sigma_{3}$ & $\pi_{1}$ & $\pi_{2}$ & $\pi_{3}$ \\
\hline \multirow[t]{24}{*}{100} & \multirow[t]{6}{*}{$(0.2 ; 0.2)$} & \multirow[t]{2}{*}{ EM } & BIAS & -0.0019 & -0.0002 & -0.0091 & -0.0017 & -0.0022 & 0.0001 & -0.0155 & -0.0113 & -0.0042 & -0.0020 & 0.0072 & -0.0052 \\
\hline & & & MSE & 0.0048 & 0.0023 & 0.0059 & 0.0022 & 0.0016 & 0.0007 & 0.0023 & 0.0025 & 0.0009 & 0.0018 & 0.0021 & 0.0034 \\
\hline & & \multirow[t]{2}{*}{ CEM } & BIAS & -0.0077 & -0.0004 & 0.0010 & -0.0004 & -0.0011 & 0.0000 & -0.0208 & -0.0204 & -0.0072 & -0.0027 & 0.0042 & -0.0016 \\
\hline & & & MSE & 0.0038 & 0.0020 & 0.0036 & 0.0018 & 0.0013 & 0.0007 & 0.0016 & 0.0017 & 0.0006 & 0.0016 & 0.0017 & 0.0027 \\
\hline & & \multirow[t]{2}{*}{ SEM } & BIAS & -0.0106 & -0.0013 & 0.0041 & -0.0015 & -0.0020 & -0.0001 & -0.0249 & -0.0230 & -0.0063 & -0.0036 & 0.0046 & -0.0010 \\
\hline & & & MSE & 0.0046 & 0.0022 & 0.0044 & 0.0018 & 0.0015 & 0.0008 & 0.0022 & 0.0022 & 0.0010 & 0.0018 & 0.0021 & 0.0035 \\
\hline & \multirow[t]{6}{*}{$(0.2 ; 0.4)$} & \multirow[t]{2}{*}{ EM } & BIAS & -0.0026 & 0.0047 & 0.0012 & 0.0002 & -0.0024 & 0.0000 & -0.0168 & -0.0043 & -0.0043 & 0.0017 & 0.0022 & -0.0038 \\
\hline & & & MSE & 0.0060 & 0.0020 & 0.0018 & 0.0008 & 0.0024 & 0.0010 & 0.0016 & 0.0006 & 0.0011 & 0.0014 & 0.0022 & 0.0022 \\
\hline & & \multirow[t]{2}{*}{ CEM } & BIAS & -0.0074 & 0.0037 & 0.0040 & 0.0004 & -0.0044 & 0.0001 & -0.0215 & -0.0081 & -0.0104 & 0.0011 & 0.0022 & -0.0032 \\
\hline & & & MSE & 0.0051 & 0.0018 & 0.0017 & 0.0008 & 0.0022 & 0.0009 & 0.0013 & 0.0005 & 0.0008 & 0.0014 & 0.0022 & 0.0021 \\
\hline & & \multirow[t]{2}{*}{ SEM } & BIAS & -0.0081 & 0.0040 & 0.0042 & 0.0002 & -0.0047 & -0.0003 & -0.0222 & -0.0080 & -0.0119 & 0.0012 & 0.0031 & -0.0042 \\
\hline & & & MSE & 0.0053 & 0.0020 & 0.0018 & 0.0008 & 0.0024 & 0.0010 & 0.0016 & 0.0006 & 0.0011 & 0.0015 & 0.0023 & 0.0022 \\
\hline & \multirow[t]{6}{*}{$(0.3 ; 0.3)$} & \multirow[t]{2}{*}{ EM } & BIAS & -0.0031 & 0.0054 & 0.0026 & 0.0030 & 0.0076 & 0.0003 & -0.0084 & -0.0104 & -0.0017 & -0.0015 & 0.0002 & 0.0013 \\
\hline & & & MSE & 0.0030 & 0.0011 & 0.0025 & 0.0013 & 0.0027 & 0.0012 & 0.0011 & 0.0011 & 0.0014 & 0.0021 & 0.0022 & 0.0024 \\
\hline & & \multirow[t]{2}{*}{ CEM } & BIAS & -0.0058 & 0.0050 & 0.0049 & 0.0018 & 0.0072 & -0.0014 & -0.0122 & -0.0126 & -0.0112 & -0.0012 & 0.0022 & -0.0010 \\
\hline & & & MSE & 0.0026 & 0.0010 & 0.0024 & 0.0013 & 0.0019 & 0.0008 & 0.0009 & 0.0009 & 0.0008 & 0.0021 & 0.0022 & 0.0022 \\
\hline & & \multirow[t]{2}{*}{ SEM } & BIAS & -0.0059 & 0.0049 & 0.0073 & 0.0017 & 0.0081 & -0.0007 & -0.0122 & -0.0142 & -0.0122 & -0.0002 & 0.0010 & -0.0009 \\
\hline & & & MSE & 0.0030 & 0.0011 & 0.0026 & 0.0013 & 0.0025 & 0.0009 & 0.0011 & 0.0011 & 0.0014 & 0.0022 & 0.0022 & 0.0024 \\
\hline & \multirow[t]{6}{*}{$(0.5 ; 0.3)$} & \multirow[t]{2}{*}{ EM } & BIAS & -0.0062 & 0.0052 & -0.0045 & 0.0023 & 0.0051 & -0.0030 & -0.0053 & -0.0088 & -0.0125 & -0.0018 & -0.0006 & 0.0024 \\
\hline & & & MSE & 0.0016 & 0.0006 & 0.0026 & 0.0012 & 0.0054 & 0.0030 & 0.0006 & 0.0015 & 0.0027 & 0.0022 & 0.0023 & 0.0021 \\
\hline & & \multirow[t]{2}{*}{ CEM } & BIAS & -0.0062 & 0.0047 & -0.0033 & 0.0021 & 0.0046 & -0.0044 & -0.0064 & -0.0099 & -0.0264 & -0.0004 & 0.0011 & -0.0008 \\
\hline & & & MSE & 0.0016 & 0.0006 & 0.0023 & 0.0011 & 0.0038 & 0.0018 & 0.0005 & 0.0011 & 0.0017 & 0.0022 & 0.0019 & 0.0019 \\
\hline & & \multirow[t]{2}{*}{ SEM } & BIAS & -0.0066 & 0.0047 & -0.0037 & 0.0020 & 0.0041 & -0.0044 & -0.0066 & -0.0093 & -0.0287 & -0.0007 & 0.0013 & -0.0006 \\
\hline & & & MSE & 0.0016 & 0.0006 & 0.0026 & 0.0012 & 0.0047 & 0.0030 & 0.0006 & 0.0014 & 0.0027 & 0.0022 & 0.0022 & 0.0021 \\
\hline
\end{tabular}




\begin{tabular}{|c|c|c|c|c|c|c|c|c|c|c|c|c|c|c|c|}
\hline \multirow[t]{24}{*}{500} & \multirow[t]{6}{*}{$(0.2 ; 0.2)$} & \multirow[t]{2}{*}{ EM } & BIAS & -0.0007 & 0.0002 & -0.0005 & 0.0009 & -0.0010 & 0.0007 & -0.0011 & -0.0031 & -0.0007 & -0.0015 & 0.0013 & 0.0002 \\
\hline & & & MSE & 0.0008 & 0.0004 & 0.0007 & 0.0004 & 0.0003 & 0.0001 & 0.0003 & 0.0003 & 0.0001 & 0.0003 & 0.0003 & 0.0005 \\
\hline & & \multirow[t]{2}{*}{ CEM } & BIAS & -0.0072 & 0.0000 & 0.0057 & 0.0010 & -0.0009 & 0.0006 & -0.0088 & -0.0104 & -0.0050 & -0.0023 & 0.0007 & 0.0016 \\
\hline & & & MSE & 0.0008 & 0.0003 & 0.0007 & 0.0003 & 0.0003 & 0.0001 & 0.0003 & 0.0003 & 0.0001 & 0.0003 & 0.0003 & 0.0005 \\
\hline & & \multirow[t]{2}{*}{ SEM } & BIAS & -0.0091 & 0.0001 & 0.0069 & 0.0007 & -0.0010 & 0.0005 & -0.0100 & -0.0109 & -0.0064 & -0.0022 & 0.0012 & 0.0010 \\
\hline & & & MSE & 0.0008 & 0.0003 & 0.0007 & 0.0004 & 0.0003 & 0.0001 & 0.0003 & 0.0004 & 0.0001 & 0.0003 & 0.0003 & 0.0005 \\
\hline & \multirow[t]{6}{*}{$(0.2 ; 0.4)$} & \multirow[t]{2}{*}{ EM } & BIAS & -0.0009 & -0.0006 & 0.0018 & 0.0004 & 0.0004 & 0.0004 & -0.0025 & -0.0014 & -0.0009 & -0.0017 & 0.0032 & -0.0015 \\
\hline & & & MSE & 0.0009 & 0.0004 & 0.0004 & 0.0001 & 0.0004 & 0.0002 & 0.0003 & 0.0001 & 0.0002 & 0.0003 & 0.0005 & 0.0005 \\
\hline & & \multirow[t]{2}{*}{ CEM } & BIAS & -0.0053 & -0.0008 & 0.0046 & 0.0003 & -0.0011 & 0.0005 & -0.0081 & -0.0051 & -0.0084 & -0.0019 & 0.0035 & -0.0015 \\
\hline & & & MSE & 0.0009 & 0.0004 & 0.0004 & 0.0001 & 0.0004 & 0.0002 & 0.0003 & 0.0001 & 0.0002 & 0.0003 & 0.0005 & 0.0006 \\
\hline & & \multirow[t]{2}{*}{ SEM } & BIAS & -0.0062 & -0.0009 & 0.0058 & 0.0001 & -0.0012 & 0.0004 & -0.0087 & -0.0058 & -0.0104 & -0.0016 & 0.0036 & -0.0020 \\
\hline & & & MSE & 0.0009 & 0.0004 & 0.0004 & 0.0001 & 0.0004 & 0.0002 & 0.0003 & 0.0001 & 0.0002 & 0.0003 & 0.0005 & 0.0006 \\
\hline & \multirow[t]{6}{*}{$(0.3 ; 0.3)$} & \multirow[t]{2}{*}{ EM } & BIAS & -0.0007 & -0.0007 & 0.0001 & -0.0009 & -0.0016 & 0.0004 & -0.0022 & -0.0015 & -0.0008 & -0.0003 & 0.0000 & 0.0004 \\
\hline & & & MSE & 0.0006 & 0.0002 & 0.0005 & 0.0002 & 0.0004 & 0.0001 & 0.0002 & 0.0002 & 0.0002 & 0.0004 & 0.0004 & 0.0005 \\
\hline & & \multirow[t]{2}{*}{ CEM } & BIAS & -0.0042 & -0.0005 & 0.0034 & -0.0010 & -0.0014 & 0.0001 & -0.0065 & -0.0057 & -0.0088 & -0.0002 & 0.0002 & 0.0000 \\
\hline & & & MSE & 0.0006 & 0.0002 & 0.0004 & 0.0002 & 0.0003 & 0.0001 & 0.0002 & 0.0002 & 0.0002 & 0.0004 & 0.0004 & 0.0005 \\
\hline & & \multirow[t]{2}{*}{ SEM } & BIAS & -0.0058 & -0.0004 & 0.0043 & -0.0007 & -0.0015 & 0.0002 & -0.0076 & -0.0065 & -0.0107 & -0.0002 & 0.0004 & -0.0002 \\
\hline & & & MSE & 0.0006 & 0.0002 & 0.0005 & 0.0002 & 0.0003 & 0.0001 & 0.0002 & 0.0002 & 0.0002 & 0.0004 & 0.0005 & 0.0005 \\
\hline & \multirow[t]{6}{*}{$(0.5 ; 0.3)$} & \multirow[t]{2}{*}{ EM } & BIAS & 0.0011 & -0.0004 & 0.0049 & -0.0019 & 0.0020 & -0.0026 & -0.0024 & -0.0017 & 0.0009 & -0.0026 & 0.0002 & 0.0024 \\
\hline & & & MSE & 0.0003 & 0.0001 & 0.0005 & 0.0002 & 0.0008 & 0.0004 & 0.0001 & 0.0002 & 0.0004 & 0.0005 & 0.0004 & 0.0004 \\
\hline & & \multirow[t]{2}{*}{ CEM } & BIAS & 0.0001 & -0.0005 & 0.0063 & -0.0017 & 0.0033 & -0.0022 & -0.0041 & -0.0041 & -0.0137 & -0.0016 & 0.0008 & 0.0007 \\
\hline & & & MSE & 0.0003 & 0.0001 & 0.0005 & 0.0002 & 0.0007 & 0.0003 & 0.0001 & 0.0002 & 0.0004 & 0.0005 & 0.0004 & 0.0004 \\
\hline & & \multirow[t]{2}{*}{ SEM } & BIAS & -0.0003 & -0.0006 & 0.0072 & -0.0018 & 0.0045 & -0.0025 & -0.0045 & -0.0047 & -0.0167 & -0.0011 & 0.0009 & 0.0002 \\
\hline & & & MSE & 0.0003 & 0.0001 & 0.0006 & 0.0002 & 0.0008 & 0.0003 & 0.0001 & 0.0002 & 0.0005 & 0.0005 & 0.0004 & 0.0004 \\
\hline
\end{tabular}


Table 12. Mean square error (MSE) and bias of estimates based on 200 replications of the three component mixtures of linear regression models when the true regression lines are parallel and the algorithms were initiated by random numbers (second strategy).

\begin{tabular}{|c|c|c|c|c|c|c|c|c|c|c|c|c|c|c|c|}
\hline$n$ & $\left(\pi_{1} ; \pi_{2}\right)$ & Algorithm & & $\beta_{10}$ & $\beta_{11}$ & $\beta_{20}$ & $\beta_{21}$ & $\beta_{30}$ & $\beta_{31}$ & $\sigma_{1}$ & $\sigma_{2}$ & $\sigma_{3}$ & $\pi_{1}$ & $\pi_{2}$ & $\pi_{3}$ \\
\hline \multirow[t]{24}{*}{100} & \multirow[t]{6}{*}{$(0.2 ; 0.2)$} & \multirow[t]{2}{*}{ EM } & BIAS & 0.2690 & 0.1378 & -0.2562 & -0.1749 & 0.0144 & -0.0117 & 0.0999 & 0.0896 & 0.0462 & 0.0826 & 0.0838 & -0.1664 \\
\hline & & & MSE & 0.1967 & 0.0896 & 0.1866 & 0.1047 & 0.0397 & 0.0541 & 0.0480 & 0.0465 & 0.0306 & 0.0444 & 0.0417 & 0.0726 \\
\hline & & \multirow[t]{2}{*}{ CEM } & BIAS & 0.4007 & 0.3706 & -0.4096 & -0.3569 & 0.0039 & -0.0226 & 0.1198 & 0.1311 & 0.0357 & 0.0843 & 0.1085 & -0.1928 \\
\hline & & & MSE & 0.2902 & 0.2789 & 0.2915 & 0.2598 & 0.0391 & 0.0823 & 0.0519 & 0.0547 & 0.0349 & 0.0509 & 0.0605 & 0.1007 \\
\hline & & \multirow[t]{2}{*}{ SEM } & BIAS & 0.0693 & 0.1317 & -0.0833 & -0.1251 & -0.0202 & -0.0125 & 0.0012 & 0.0193 & 0.0104 & 0.0178 & 0.0420 & -0.0597 \\
\hline & & & MSE & 0.0422 & 0.0910 & 0.0543 & 0.0843 & 0.0208 & 0.0323 & 0.0106 & 0.0128 & 0.0092 & 0.0106 & 0.0169 & 0.0253 \\
\hline & \multirow[t]{6}{*}{$(0.2 ; 0.4)$} & \multirow[t]{2}{*}{ EM } & BIAS & 0.2601 & 0.1257 & 0.0041 & -0.0552 & 0.0962 & -0.0676 & 0.0973 & -0.0122 & 0.0462 & 0.0814 & -0.0471 & -0.0343 \\
\hline & & & MSE & 0.1805 & 0.0747 & 0.0178 & 0.0301 & 0.1000 & 0.0652 & 0.0422 & 0.0052 & 0.0351 & 0.0339 & 0.0114 & 0.0240 \\
\hline & & \multirow[t]{2}{*}{ CEM } & BIAS & 0.4535 & 0.4104 & -0.2463 & -0.4338 & 0.1676 & 0.0216 & 0.1548 & 0.1351 & 0.0549 & 0.1326 & -0.0589 & -0.0737 \\
\hline & & & MSE & 0.3557 & 0.2697 & 0.1399 & 0.2777 & 0.0937 & 0.1257 & 0.0593 & 0.0567 & 0.0367 & 0.0487 & 0.0333 & 0.0383 \\
\hline & & \multirow[t]{2}{*}{ SEM } & BIAS & 0.0688 & 0.1139 & -0.0410 & -0.1149 & 0.0321 & -0.0013 & 0.0115 & 0.0209 & 0.0238 & 0.0326 & -0.0006 & -0.0320 \\
\hline & & & MSE & 0.0460 & 0.0621 & 0.0154 & 0.0536 & 0.0411 & 0.0609 & 0.0108 & 0.0085 & 0.0106 & 0.0136 & 0.0105 & 0.0171 \\
\hline & \multirow[t]{6}{*}{$(0.3 ; 0.3)$} & \multirow[t]{2}{*}{ EM } & BIAS & 0.1462 & 0.1734 & -0.1253 & -0.1087 & -0.0050 & -0.0204 & 0.0653 & 0.0533 & 0.0203 & 0.0196 & 0.0103 & -0.0298 \\
\hline & & & MSE & 0.0866 & 0.0914 & 0.0843 & 0.0648 & 0.0914 & 0.0980 & 0.0311 & 0.0295 & 0.0226 & 0.0215 & 0.0186 & 0.0283 \\
\hline & & \multirow[t]{2}{*}{ CEM } & BIAS & 0.4101 & 0.4673 & -0.4270 & -0.4611 & -0.0066 & 0.0369 & 0.1524 & 0.1823 & 0.0635 & 0.0191 & 0.0399 & -0.0589 \\
\hline & & & MSE & 0.2822 & 0.3335 & 0.2958 & 0.3587 & 0.0564 & 0.1103 & 0.0664 & 0.0817 & 0.0470 & 0.0316 & 0.0358 & 0.0415 \\
\hline & & \multirow[t]{2}{*}{ SEM } & BIAS & 0.0532 & 0.1942 & -0.0452 & -0.1498 & -0.0107 & -0.0149 & 0.0056 & 0.0182 & 0.0651 & -0.0101 & 0.0124 & -0.0023 \\
\hline & & & MSE & 0.0195 & 0.1029 & 0.0256 & 0.0728 & 0.0275 & 0.0738 & 0.0087 & 0.0084 & 0.0177 & 0.0093 & 0.0075 & 0.0146 \\
\hline & \multirow[t]{6}{*}{$(0.5 ; 0.3)$} & \multirow[t]{2}{*}{ EM } & BIAS & -0.0127 & 0.0400 & -0.1338 & -0.0909 & -0.1181 & 0.1131 & -0.0122 & 0.0901 & 0.0580 & -0.0538 & 0.0215 & 0.0322 \\
\hline & & & MSE & 0.0071 & 0.0287 & 0.0588 & 0.0452 & 0.1599 & 0.0950 & 0.0036 & 0.0357 & 0.0320 & 0.0147 & 0.0102 & 0.0147 \\
\hline & & \multirow[t]{2}{*}{ CEM } & BIAS & 0.1952 & 0.4368 & -0.4128 & -0.5023 & -0.1504 & 0.0680 & 0.1353 & 0.1631 & 0.0676 & -0.1215 & 0.0478 & 0.0737 \\
\hline & & & MSE & 0.1852 & 0.2905 & 0.3023 & 0.3470 & 0.1254 & 0.2044 & 0.0704 & 0.0738 & 0.0503 & 0.0514 & 0.0396 & 0.0378 \\
\hline & & \multirow[t]{2}{*}{ SEM } & BIAS & 0.0007 & 0.0340 & -0.0540 & -0.1054 & 0.0073 & 0.0656 & -0.0026 & 0.0229 & 0.0174 & -0.0248 & -0.0073 & 0.0320 \\
\hline & & & MSE & 0.0047 & 0.0123 & 0.0195 & 0.0452 & 0.0504 & 0.0668 & 0.0043 & 0.0107 & 0.0153 & 0.0091 & 0.0068 & 0.0148 \\
\hline
\end{tabular}




\begin{tabular}{|c|c|c|c|c|c|c|c|c|c|c|c|c|c|c|c|}
\hline \multirow[t]{24}{*}{500} & \multirow[t]{6}{*}{$(0.2 ; 0.2)$} & \multirow[t]{2}{*}{ EM } & BIAS & 0.2141 & 0.0728 & -0.2771 & -0.0710 & -0.0276 & 0.0332 & 0.0862 & 0.1229 & 0.0945 & 0.0442 & 0.1010 & -0.1452 \\
\hline & & & MSE & 0.1729 & 0.0389 & 0.2200 & 0.0322 & 0.0336 & 0.0354 & 0.0406 & 0.0517 & 0.0428 & 0.0317 & 0.0581 & 0.0712 \\
\hline & & \multirow[t]{2}{*}{ CEM } & BIAS & 0.3523 & 0.3741 & -0.3525 & -0.3488 & 0.0105 & 0.0033 & 0.1241 & 0.1330 & 0.0054 & 0.0817 & 0.0833 & -0.1649 \\
\hline & & & MSE & 0.2624 & 0.2657 & 0.2504 & 0.2565 & 0.0896 & 0.0282 & 0.0702 & 0.0783 & 0.0429 & 0.0919 & 0.0865 & 0.1186 \\
\hline & & \multirow[t]{2}{*}{ SEM } & BIAS & 0.0652 & 0.1390 & -0.0701 & -0.1510 & 0.0016 & 0.0080 & 0.0322 & 0.0360 & 0.0102 & 0.0304 & 0.0311 & -0.0615 \\
\hline & & & MSE & 0.0325 & 0.0844 & 0.0338 & 0.0949 & 0.0078 & 0.0264 & 0.0117 & 0.0121 & 0.0042 & 0.0105 & 0.0078 & 0.0202 \\
\hline & \multirow[t]{6}{*}{$(0.2 ; 0.4)$} & \multirow[t]{2}{*}{ EM } & BIAS & 0.1782 & 0.0418 & 0.0099 & 0.0007 & 0.0072 & -0.0741 & 0.0920 & -0.0089 & 0.0547 & 0.0623 & -0.0154 & -0.0469 \\
\hline & & & MSE & 0.1279 & 0.0124 & 0.0006 & 0.0002 & 0.0302 & 0.0405 & 0.0362 & 0.0003 & 0.0237 & 0.0257 & 0.0016 & 0.0189 \\
\hline & & \multirow[t]{2}{*}{ CEM } & BIAS & 0.3918 & 0.4666 & -0.2647 & -0.5511 & 0.1291 & 0.0571 & 0.1782 & 0.1696 & 0.0434 & 0.1226 & -0.0674 & -0.0553 \\
\hline & & & MSE & 0.2850 & 0.2792 & 0.1236 & 0.3637 & 0.0614 & 0.0804 & 0.0721 & 0.0590 & 0.0370 & 0.0464 & 0.0359 & 0.0424 \\
\hline & & \multirow[t]{2}{*}{ SEM } & BIAS & 0.0740 & 0.1050 & -0.0724 & -0.1717 & 0.0349 & 0.0664 & 0.0231 & 0.0560 & 0.0460 & 0.0323 & 0.0132 & -0.0454 \\
\hline & & & MSE & 0.0449 & 0.0545 & 0.0245 & 0.0762 & 0.0367 & 0.0941 & 0.0117 & 0.0136 & 0.0174 & 0.0165 & 0.0126 & 0.0253 \\
\hline & \multirow{6}{*}{$(0.3 ; 0.3)$} & \multirow[t]{2}{*}{ EM } & BIAS & 0.2237 & 0.1193 & -0.1993 & -0.1136 & 0.0128 & -0.0167 & 0.1187 & 0.0937 & 0.0984 & 0.0175 & -0.0028 & -0.0148 \\
\hline & & & MSE & 0.1473 & 0.0671 & 0.1452 & 0.0620 & 0.0645 & 0.0626 & 0.0561 & 0.0536 & 0.0211 & 0.0172 & 0.0211 & 0.0176 \\
\hline & & \multirow[t]{2}{*}{ CEM } & BIAS & 0.3209 & 0.6193 & -0.3528 & -0.6077 & 0.0111 & 0.0245 & 0.2382 & 0.2761 & -0.0418 & 0.0341 & 0.0656 & -0.0997 \\
\hline & & & MSE & 0.1439 & 0.4273 & 0.1622 & 0.4097 & 0.0244 & 0.0236 & 0.0815 & 0.1010 & 0.0196 & 0.0157 & 0.0233 & 0.0267 \\
\hline & & \multirow[t]{2}{*}{ SEM } & BIAS & 0.0888 & 0.1914 & -0.1096 & -0.2279 & 0.0329 & 0.0254 & 0.0677 & 0.0626 & 0.0623 & 0.0355 & 0.0237 & -0.0592 \\
\hline & & & MSE & 0.0385 & 0.0914 & 0.0424 & 0.1166 & 0.0396 & 0.1041 & 0.0188 & 0.0168 & 0.0172 & 0.0123 & 0.0112 & 0.0187 \\
\hline & \multirow[t]{6}{*}{$(0.5 ; 0.3)$} & \multirow[t]{2}{*}{ EM } & BIAS & -0.0081 & 0.0096 & -0.0757 & -0.0629 & 0.0511 & 0.1515 & -0.0069 & 0.0636 & 0.0277 & -0.0096 & 0.0073 & 0.0093 \\
\hline & & & MSE & 0.0040 & 0.0048 & 0.0223 & 0.0455 & 0.0352 & 0.0335 & 0.0043 & 0.0220 & 0.0135 & 0.0044 & 0.0083 & 0.0157 \\
\hline & & \multirow[t]{2}{*}{ CEM } & BIAS & 0.1925 & 0.5073 & -0.4631 & -0.4320 & -0.1859 & 0.0683 & 0.0919 & 0.1796 & 0.1047 & -0.1975 & 0.0750 & 0.1225 \\
\hline & & & MSE & 0.1766 & 0.3299 & 0.4705 & 0.2687 & 0.1391 & 0.1831 & 0.0889 & 0.1335 & 0.1212 & 0.1207 & 0.1056 & 0.1390 \\
\hline & & \multirow[t]{2}{*}{ SEM } & BIAS & 0.0080 & 0.0150 & -0.0724 & -0.1187 & 0.0579 & 0.1196 & 0.0070 & 0.0667 & -0.0003 & -0.0053 & 0.0070 & -0.0017 \\
\hline & & & MSE & 0.0033 & 0.0053 & 0.0207 & 0.0430 & 0.0363 & 0.0747 & 0.0035 & 0.0201 & 0.0127 & 0.0032 & 0.0087 & 0.0114 \\
\hline
\end{tabular}


Table 13. Mean square error and bias of estimates based on 200 replications of the three-component mixtures of linear regression models when the true regression lines are concurrent and the true values are used as the starting values.

\begin{tabular}{|c|c|c|c|c|c|c|c|c|c|c|c|c|c|c|c|}
\hline$n$ & $\left(\pi_{1} ; \pi_{2}\right)$ & Algorithm & & $\beta_{10}$ & $\beta_{11}$ & $\beta_{20}$ & $\beta_{21}$ & $\beta_{30}$ & $\beta_{31}$ & $\sigma_{1}$ & $\sigma_{2}$ & $\sigma_{3}$ & $\pi_{1}$ & $\pi_{2}$ & $\pi_{3}$ \\
\hline \multirow[t]{24}{*}{100} & \multirow[t]{6}{*}{$(0.2 ; 0.2)$} & \multirow[t]{2}{*}{ EM } & BIAS & 0.0000 & -0.0140 & 0.0936 & -0.0352 & -0.0004 & 0.0004 & -0.0423 & -0.1340 & -0.0054 & 0.0013 & -0.0072 & 0.0059 \\
\hline & & & MSE & 0.0311 & 0.0211 & 0.2348 & 0.0842 & 0.0031 & 0.0013 & 0.0145 & 0.0854 & 0.0013 & 0.0025 & 0.0035 & 0.0035 \\
\hline & & \multirow[t]{2}{*}{ CEM } & BIAS & -0.0087 & 0.0057 & 0.2308 & -0.1876 & -0.0040 & 0.0021 & -0.0673 & -0.1756 & -0.0083 & 0.0249 & -0.0595 & 0.0346 \\
\hline & & & MSE & 0.0287 & 0.0142 & 0.3238 & 0.1339 & 0.0030 & 0.0012 & 0.0147 & 0.0990 & 0.0012 & 0.0034 & 0.0062 & 0.0044 \\
\hline & & \multirow[t]{2}{*}{ SEM } & BIAS & -0.0043 & -0.0258 & 0.1674 & -0.1268 & 0.0006 & 0.0023 & -0.0672 & -0.1988 & -0.0156 & 0.0022 & -0.0070 & 0.0040 \\
\hline & & & MSE & 0.0351 & 0.0294 & 0.4003 & 0.1683 & 0.0049 & 0.0013 & 0.0270 & 0.1289 & 0.0017 & 0.0034 & 0.0052 & 0.0051 \\
\hline & \multirow{6}{*}{$(0.2 ; 0.4)$} & \multirow[t]{2}{*}{ EM } & BIAS & -0.0025 & 0.0034 & 0.0404 & -0.0240 & -0.0019 & -0.0011 & -0.0530 & -0.0649 & -0.0135 & 0.0034 & -0.0052 & 0.0018 \\
\hline & & & MSE & 0.0286 & 0.0182 & 0.0578 & 0.0291 & 0.0065 & 0.0023 & 0.0158 & 0.0256 & 0.0026 & 0.0026 & 0.0044 & 0.0029 \\
\hline & & \multirow[t]{2}{*}{ CEM } & BIAS & -0.0216 & 0.0239 & 0.1354 & -0.1244 & -0.0078 & 0.0035 & -0.0982 & -0.1134 & -0.0310 & 0.0151 & -0.0519 & 0.0368 \\
\hline & & & MSE & 0.0276 & 0.0180 & 0.0792 & 0.0472 & 0.0065 & 0.0024 & 0.0194 & 0.0357 & 0.0027 & 0.0050 & 0.0092 & 0.0045 \\
\hline & & \multirow[t]{2}{*}{ SEM } & BIAS & -0.0332 & 0.0106 & 0.0998 & -0.0877 & -0.0049 & 0.0029 & -0.1035 & -0.1010 & -0.0332 & -0.0023 & 0.0041 & -0.0019 \\
\hline & & & MSE & 0.0320 & 0.0401 & 0.0909 & 0.0560 & 0.0089 & 0.0029 & 0.0255 & 0.0442 & 0.0035 & 0.0040 & 0.0073 & 0.0035 \\
\hline & \multirow{6}{*}{$(0.3 ; 0.3)$} & \multirow[t]{2}{*}{ EM } & BIAS & -0.0058 & -0.0087 & 0.0140 & 0.0035 & -0.0006 & 0.0001 & -0.0207 & -0.0806 & -0.0113 & 0.0041 & -0.0047 & 0.0006 \\
\hline & & & MSE & 0.0176 & 0.0103 & 0.1046 & 0.0497 & 0.0058 & 0.0024 & 0.0080 & 0.0380 & 0.0020 & 0.0028 & 0.0044 & 0.0031 \\
\hline & & \multirow[t]{2}{*}{ CEM } & BIAS & -0.0152 & 0.0118 & 0.1509 & -0.1375 & -0.0040 & 0.0025 & -0.0550 & -0.1166 & -0.0213 & 0.0432 & -0.0789 & 0.0357 \\
\hline & & & MSE & 0.0175 & 0.0096 & 0.1828 & 0.0836 & 0.0058 & 0.0023 & 0.0095 & 0.0490 & 0.0020 & 0.0053 & 0.0100 & 0.0042 \\
\hline & & \multirow[t]{2}{*}{ SEM } & BIAS & -0.0243 & 0.0057 & 0.1019 & -0.0883 & -0.0006 & 0.0032 & -0.0548 & -0.1298 & -0.0296 & -0.0003 & 0.0036 & -0.0033 \\
\hline & & & MSE & 0.0167 & 0.0144 & 0.1802 & 0.0983 & 0.0081 & 0.0031 & 0.0124 & 0.0569 & 0.0033 & 0.0039 & 0.0073 & 0.0041 \\
\hline & \multirow[t]{6}{*}{$(0.5 ; 0.3)$} & \multirow[t]{2}{*}{ EM } & BIAS & 0.0075 & -0.0058 & 0.0206 & -0.0322 & 0.0001 & 0.0005 & -0.0180 & -0.0940 & -0.0249 & 0.0055 & -0.0097 & 0.0043 \\
\hline & & & MSE & 0.0125 & 0.0045 & 0.1008 & 0.0578 & 0.0205 & 0.0068 & 0.0040 & 0.0481 & 0.0052 & 0.0036 & 0.0045 & 0.0022 \\
\hline & & \multirow[t]{2}{*}{ CEM } & BIAS & 0.0031 & 0.0013 & 0.1642 & -0.1778 & -0.0032 & 0.0037 & -0.0365 & -0.1304 & -0.0426 & 0.0579 & -0.0866 & 0.0287 \\
\hline & & & MSE & 0.0127 & 0.0046 & 0.1213 & 0.1031 & 0.0149 & 0.0054 & 0.0051 & 0.0577 & 0.0052 & 0.0064 & 0.0109 & 0.0032 \\
\hline & & \multirow[t]{2}{*}{ SEM } & BIAS & -0.0057 & 0.0048 & 0.1294 & -0.1313 & -0.0012 & 0.0036 & -0.0448 & -0.1403 & -0.0481 & 0.0088 & -0.0116 & 0.0028 \\
\hline & & & MSE & 0.0124 & 0.0063 & 0.1265 & 0.1141 & 0.0312 & 0.0091 & 0.0066 & 0.0666 & 0.0078 & 0.0045 & 0.0063 & 0.0029 \\
\hline
\end{tabular}




\begin{tabular}{|c|c|c|c|c|c|c|c|c|c|c|c|c|c|c|c|}
\hline \multirow[t]{24}{*}{500} & \multirow[t]{6}{*}{$(0.2 ; 0.2)$} & \multirow[t]{2}{*}{ EM } & BIAS & -0.0002 & -0.0043 & 0.0001 & -0.0052 & -0.0015 & 0.0000 & -0.0025 & -0.0234 & -0.0008 & 0.0016 & -0.0023 & 0.0007 \\
\hline & & & MSE & 0.0050 & 0.0025 & 0.0323 & 0.0115 & 0.0007 & 0.0003 & 0.0021 & 0.0107 & 0.0002 & 0.0004 & 0.0007 & 0.0006 \\
\hline & & \multirow[t]{2}{*}{ CEM } & BIAS & -0.0039 & 0.0090 & 0.1863 & -0.2079 & -0.0031 & 0.0005 & -0.0321 & -0.0747 & -0.0023 & 0.0352 & -0.0684 & 0.0332 \\
\hline & & & MSE & 0.0053 & 0.0024 & 0.0742 & 0.0586 & 0.0007 & 0.0003 & 0.0033 & 0.0197 & 0.0002 & 0.0018 & 0.0053 & 0.0016 \\
\hline & & \multirow[t]{2}{*}{ SEM } & BIAS & -0.0184 & 0.0196 & 0.1459 & -0.1632 & -0.0054 & 0.0036 & -0.0350 & -0.0787 & -0.0117 & 0.0029 & -0.0057 & 0.0028 \\
\hline & & & MSE & 0.0059 & 0.0033 & 0.0594 & 0.0425 & 0.0008 & 0.0003 & 0.0037 & 0.0198 & 0.0003 & 0.0005 & 0.0009 & 0.0007 \\
\hline & \multirow[t]{6}{*}{$(0.2 ; 0.4)$} & \multirow[t]{2}{*}{ EM } & BIAS & -0.0021 & -0.0005 & 0.0018 & -0.0050 & 0.0037 & -0.0033 & -0.0072 & -0.0111 & -0.0017 & 0.0006 & -0.0016 & 0.0010 \\
\hline & & & MSE & 0.0042 & 0.0034 & 0.0154 & 0.0072 & 0.0012 & 0.0004 & 0.0025 & 0.0041 & 0.0003 & 0.0004 & 0.0008 & 0.0006 \\
\hline & & \multirow[t]{2}{*}{ CEM } & BIAS & -0.0209 & 0.0343 & 0.1263 & -0.1387 & -0.0027 & 0.0012 & -0.0659 & -0.0610 & -0.0180 & 0.0246 & -0.0686 & 0.0440 \\
\hline & & & MSE & 0.0050 & 0.0049 & 0.0328 & 0.0273 & 0.0013 & 0.0005 & 0.0071 & 0.0094 & 0.0006 & 0.0022 & 0.0071 & 0.0026 \\
\hline & & \multirow[t]{2}{*}{ SEM } & BIAS & -0.0272 & 0.0298 & 0.1079 & -0.1147 & -0.0029 & 0.0031 & -0.0477 & -0.0577 & -0.0222 & 0.0012 & -0.0013 & 0.0001 \\
\hline & & & MSE & 0.0054 & 0.0059 & 0.0291 & 0.0220 & 0.0016 & 0.0005 & 0.0047 & 0.0087 & 0.0008 & 0.0005 & 0.0012 & 0.0007 \\
\hline & \multirow{6}{*}{$(0.3 ; 0.3)$} & \multirow[t]{2}{*}{ EM } & BIAS & 0.0003 & -0.0011 & -0.0126 & 0.0047 & 0.0031 & 0.0018 & -0.0104 & -0.0171 & -0.0021 & -0.0027 & 0.0010 & 0.0017 \\
\hline & & & MSE & 0.0030 & 0.0020 & 0.0185 & 0.0081 & 0.0011 & 0.0004 & 0.0015 & 0.0046 & 0.0003 & 0.0007 & 0.0009 & 0.0006 \\
\hline & & \multirow[t]{2}{*}{ CEM } & BIAS & -0.0058 & 0.0128 & 0.1562 & -0.1741 & -0.0021 & 0.0048 & -0.0442 & -0.0628 & -0.0111 & 0.0454 & -0.0859 & 0.0405 \\
\hline & & & MSE & 0.0031 & 0.0021 & 0.0466 & 0.0398 & 0.0012 & 0.0004 & 0.0036 & 0.0104 & 0.0005 & 0.0029 & 0.0083 & 0.0023 \\
\hline & & \multirow[t]{2}{*}{ SEM } & BIAS & -0.0188 & 0.0211 & 0.1208 & -0.1329 & -0.0046 & 0.0082 & -0.0444 & -0.0680 & -0.0182 & -0.0011 & -0.0017 & 0.0028 \\
\hline & & & MSE & 0.0035 & 0.0027 & 0.0375 & 0.0279 & 0.0014 & 0.0005 & 0.0036 & 0.0109 & 0.0007 & 0.0009 & 0.0012 & 0.0007 \\
\hline & \multirow{6}{*}{$(0.5 ; 0.3)$} & \multirow[t]{2}{*}{$\mathrm{EM}$} & BIAS & 0.0049 & -0.0046 & -0.0076 & 0.0026 & -0.0028 & 0.0008 & -0.0035 & -0.0113 & -0.0048 & -0.0014 & -0.0007 & 0.0021 \\
\hline & & & MSE & 0.0024 & 0.0012 & 0.0165 & 0.0075 & 0.0027 & 0.0009 & 0.0008 & 0.0057 & 0.0007 & 0.0007 & 0.0007 & 0.0004 \\
\hline & & \multirow[t]{2}{*}{ CEM } & BIAS & 0.0023 & 0.0032 & 0.1634 & -0.1818 & -0.0118 & 0.0064 & -0.0223 & -0.0582 & -0.0234 & 0.0590 & -0.0916 & 0.0326 \\
\hline & & & MSE & 0.0023 & 0.0011 & 0.0442 & 0.0424 & 0.0027 & 0.0010 & 0.0013 & 0.0109 & 0.0012 & 0.0042 & 0.0092 & 0.0016 \\
\hline & & \multirow[t]{2}{*}{ SEM } & BIAS & -0.0102 & 0.0132 & 0.1257 & -0.1384 & -0.0145 & 0.0103 & -0.0329 & -0.0627 & -0.0255 & -0.0007 & -0.0014 & 0.0021 \\
\hline & & & MSE & 0.0026 & 0.0015 & 0.0338 & 0.0299 & 0.0037 & 0.0013 & 0.0019 & 0.0116 & 0.0015 & 0.0009 & 0.0011 & 0.0005 \\
\hline
\end{tabular}


Table 14. Mean square error and bias of estimates based on 200 replications of the three-component mixtures of linear regression models when the true regression lines are concurrent and the algorithms are initiated by random numbers (second strategy).

\begin{tabular}{|c|c|c|c|c|c|c|c|c|c|c|c|c|c|c|c|}
\hline$n$ & $\left(\pi_{1} ; \pi_{2}\right)$ & Algorithm & & $\beta_{10}$ & $\beta_{11}$ & $\beta_{20}$ & $\beta_{21}$ & $\beta_{30}$ & $\beta_{31}$ & $\sigma_{1}$ & $\sigma_{2}$ & $\sigma_{3}$ & $\pi_{1}$ & $\pi_{2}$ & $\pi_{3}$ \\
\hline \multirow[t]{24}{*}{100} & \multirow[t]{6}{*}{$(0.2 ; 0.2)$} & \multirow[t]{2}{*}{ EM } & BIAS & 0.8400 & -0.3784 & -0.6563 & 0.6721 & 0.1151 & -0.0583 & 0.5173 & 0.0480 & -0.0276 & 0.0927 & 0.0462 & -0.1360 \\
\hline & & & MSE & 1.7077 & 0.5013 & 2.5002 & 0.8845 & 0.0892 & 0.0418 & 0.5711 & 0.2904 & 0.0175 & 0.0154 & 0.0158 & 0.0532 \\
\hline & & \multirow[t]{2}{*}{ CEM } & BIAS & 0.3162 & 0.0866 & -1.4930 & 1.1180 & -0.0275 & -0.0902 & 0.2751 & -0.3033 & 0.1084 & 0.0613 & 0.0645 & -0.0831 \\
\hline & & & MSE & 1.0811 & 1.2343 & 4.8535 & 1.6602 & 0.8241 & 0.1960 & 0.1930 & 0.2901 & 0.0538 & 0.0150 & 0.0398 & 0.0457 \\
\hline & & \multirow[t]{2}{*}{ SEM } & BIAS & 0.1256 & -0.1545 & -0.0477 & 0.1817 & 0.0841 & 0.0019 & 0.0636 & -0.2725 & -0.0224 & 0.0235 & -0.0049 & -0.0305 \\
\hline & & & MSE & 0.1664 & 0.1221 & 1.5723 & 0.4671 & 0.1458 & 0.0181 & 0.0758 & 0.1994 & 0.0052 & 0.0050 & 0.0075 & 0.0230 \\
\hline & \multirow[t]{6}{*}{$(0.2 ; 0.4)$} & \multirow[t]{2}{*}{ EM } & BIAS & 0.3883 & 0.4888 & -0.0456 & 0.0282 & -0.1005 & -0.5136 & 0.1745 & -0.1304 & 0.1590 & 0.0358 & -0.0206 & -0.0104 \\
\hline & & & MSE & 0.5338 & 0.8482 & 0.4567 & 0.0898 & 0.3316 & 0.9316 & 0.1347 & 0.1027 & 0.1380 & 0.0095 & 0.0185 & 0.0133 \\
\hline & & \multirow[t]{2}{*}{ CEM } & BIAS & 0.7816 & -0.4169 & -1.3077 & 1.0999 & 0.4376 & -0.3247 & 0.4434 & -0.4823 & 0.2841 & 0.1855 & -0.1507 & 0.0555 \\
\hline & & & MSE & 1.2050 & 0.5358 & 2.9933 & 1.3721 & 0.7384 & 0.2402 & 0.2709 & 0.4641 & 0.1131 & 0.0503 & 0.0614 & 0.0186 \\
\hline & & \multirow[t]{2}{*}{ SEM } & BIAS & 0.1076 & -0.1517 & 0.0390 & 0.0461 & 0.0053 & -0.0454 & -0.0155 & -0.1740 & -0.0008 & 0.0105 & -0.0050 & -0.0033 \\
\hline & & & MSE & 0.2502 & 0.2632 & 0.2890 & 0.1060 & 0.1840 & 0.1066 & 0.0649 & 0.1018 & 0.0151 & 0.0081 & 0.0138 & 0.0051 \\
\hline & \multirow[t]{6}{*}{$(0.3 ; 0.3)$} & \multirow[t]{2}{*}{ EM } & BIAS & 0.3689 & 0.8037 & -0.0467 & 0.1022 & -0.3519 & -0.6896 & 0.2695 & -0.1885 & 0.3123 & 0.0057 & 0.0110 & -0.0136 \\
\hline & & & MSE & 0.3507 & 1.1933 & 0.8121 & 0.1036 & 0.7812 & 1.2772 & 0.1756 & 0.1669 & 0.3192 & 0.0073 & 0.0239 & 0.0198 \\
\hline & & \multirow[t]{2}{*}{ CEM } & BIAS & 0.1865 & -0.0945 & -1.1895 & 1.0374 & -0.0569 & -0.1776 & 0.2257 & -0.5097 & 0.2093 & 0.0786 & -0.0520 & 0.0108 \\
\hline & & & MSE & 1.2765 & 1.4306 & 4.5278 & 2.3426 & 1.0037 & 0.3010 & 0.1219 & 0.4194 & 0.0968 & 0.0219 & 0.0430 & 0.0255 \\
\hline & & \multirow[t]{2}{*}{ SEM } & BIAS & 0.0275 & 0.0028 & 0.0466 & 0.0635 & -0.0570 & -0.0437 & -0.0002 & -0.1746 & -0.0111 & 0.0077 & 0.0056 & -0.0087 \\
\hline & & & MSE & 0.0494 & 0.1469 & 0.4525 & 0.2202 & 0.2087 & 0.1012 & 0.0356 & 0.1077 & 0.0123 & 0.0065 & 0.0137 & 0.0064 \\
\hline & \multirow[t]{6}{*}{$(0.5 ; 0.3)$} & \multirow[t]{2}{*}{ EM } & BIAS & 0.0104 & 0.2160 & -0.1656 & 0.1543 & -0.9677 & -0.0196 & 0.0517 & -0.1317 & 0.2148 & -0.0608 & 0.0107 & 0.0405 \\
\hline & & & MSE & 0.0606 & 0.3546 & 0.9584 & 0.2328 & 3.2270 & 0.8713 & 0.0834 & 0.1161 & 0.1963 & 0.0195 & 0.0150 & 0.0143 \\
\hline & & \multirow{2}{*}{ CEM } & BIAS & -0.1169 & 0.1809 & -0.7621 & 0.5725 & -0.0973 & -0.3517 & 0.0941 & -0.3637 & 0.2928 & -0.0892 & -0.0030 & 0.1005 \\
\hline & & & MSE & 1.7416 & 1.5340 & 1.1423 & 1.0504 & 1.9708 & 0.8847 & 0.0827 & 0.2958 & 0.2242 & 0.0381 & 0.0389 & 0.0396 \\
\hline & & \multirow[t]{2}{*}{ SEM } & BIAS & 0.0025 & 0.0497 & 0.0017 & 0.0673 & -0.2640 & -0.0401 & -0.0211 & -0.1860 & 0.0146 & -0.0185 & 0.0048 & 0.0114 \\
\hline & & & MSE & 0.0263 & 0.1327 & 0.5147 & 0.2810 & 1.2569 & 0.3061 & 0.0287 & 0.1249 & 0.0404 & 0.0113 & 0.0140 & 0.0090 \\
\hline
\end{tabular}




\begin{tabular}{|c|c|c|c|c|c|c|c|c|c|c|c|c|c|c|c|}
\hline \multirow[t]{24}{*}{500} & \multirow[t]{6}{*}{$(0.2 ; 0.2)$} & \multirow[t]{2}{*}{ EM } & BIAS & 0.6484 & -0.3058 & -0.5384 & 0.4023 & 0.1225 & -0.0523 & 0.4127 & 0.1709 & -0.0283 & 0.0724 & 0.0500 & -0.1238 \\
\hline & & & MSE & 1.2694 & 0.2997 & 1.3626 & 0.4702 & 0.0843 & 0.0225 & 0.4876 & 0.1897 & 0.0056 & 0.0136 & 0.0140 & 0.0603 \\
\hline & & \multirow[t]{2}{*}{ CEM } & BIAS & 0.3020 & -0.1519 & -1.9050 & 1.3952 & 0.0174 & -0.1059 & 0.3382 & -0.4906 & 0.1945 & 0.0950 & -0.0383 & 0.0444 \\
\hline & & & MSE & 0.3414 & 0.1973 & 5.6680 & 2.2912 & 0.2873 & 0.1283 & 0.1601 & 0.4391 & 0.0616 & 0.0128 & 0.0304 & 0.0181 \\
\hline & & \multirow[t]{2}{*}{ SEM } & BIAS & -0.0057 & -0.0321 & 0.1549 & -0.0948 & -0.0057 & 0.0039 & -0.0105 & -0.0992 & -0.0126 & 0.0039 & -0.0036 & -0.0003 \\
\hline & & & MSE & 0.0106 & 0.0263 & 0.0841 & 0.0720 & 0.0009 & 0.0003 & 0.0117 & 0.0308 & 0.0004 & 0.0008 & 0.0012 & 0.0007 \\
\hline & \multirow[t]{6}{*}{$(0.2 ; 0.4)$} & \multirow[t]{2}{*}{ EM } & BIAS & 0.1769 & 0.2390 & -0.0815 & 0.0215 & -0.0044 & -0.3033 & 0.1006 & -0.0328 & 0.0972 & 0.0212 & -0.0146 & -0.0030 \\
\hline & & & MSE & 0.2048 & 0.3590 & 0.2024 & 0.0175 & 0.0673 & 0.5136 & 0.0638 & 0.0272 & 0.0724 & 0.0040 & 0.0084 & 0.0072 \\
\hline & & \multirow[t]{2}{*}{ CEM } & BIAS & 0.4454 & -0.3176 & -1.2983 & 1.2251 & 0.2166 & -0.2296 & 0.4112 & -0.7241 & 0.3607 & 0.1802 & -0.2708 & 0.1344 \\
\hline & & & MSE & 0.5961 & 0.3753 & 2.7030 & 1.6296 & 0.3928 & 0.1471 & 0.2128 & 0.6781 & 0.1542 & 0.0413 & 0.1103 & 0.0316 \\
\hline & & \multirow[t]{2}{*}{ SEM } & BIAS & 0.0040 & -0.0101 & 0.0710 & -0.0741 & 0.0090 & -0.0268 & -0.0200 & -0.0800 & -0.0129 & 0.0033 & -0.0041 & 0.0009 \\
\hline & & & MSE & 0.0592 & 0.0720 & 0.0719 & 0.0275 & 0.0084 & 0.0517 & 0.0246 & 0.0236 & 0.0051 & 0.0020 & 0.0032 & 0.0009 \\
\hline & \multirow[t]{6}{*}{$(0.3 ; 0.3)$} & \multirow[t]{2}{*}{ EM } & BIAS & 0.2647 & 0.7148 & -0.3390 & 0.0915 & -0.1406 & -0.6479 & 0.2439 & -0.0868 & 0.3197 & 0.0090 & -0.0115 & 0.0025 \\
\hline & & & MSE & 0.2978 & 1.0441 & 0.6462 & 0.0309 & 0.4810 & 1.2732 & 0.1505 & 0.0789 & 0.2459 & 0.0047 & 0.0203 & 0.0128 \\
\hline & & \multirow[t]{2}{*}{ CEM } & BIAS & 0.2465 & -0.0609 & -1.4980 & 1.3054 & -0.0015 & -0.1812 & 0.3052 & -0.6441 & 0.2977 & 0.1188 & -0.1293 & 0.0804 \\
\hline & & & MSE & 0.1034 & 0.2484 & 3.6096 & 2.0093 & 0.5945 & 0.2428 & 0.1159 & 0.5804 & 0.1292 & 0.0201 & 0.0671 & 0.0279 \\
\hline & & \multirow[t]{2}{*}{ SEM } & BIAS & 0.0097 & 0.0851 & 0.0657 & -0.1101 & 0.0047 & -0.0681 & -0.0198 & -0.0912 & 0.0026 & 0.0001 & 0.0001 & -0.0001 \\
\hline & & & MSE & 0.0206 & 0.0997 & 0.1144 & 0.0325 & 0.0958 & 0.1430 & 0.0151 & 0.0297 & 0.0104 & 0.0013 & 0.0038 & 0.0022 \\
\hline & \multirow[t]{6}{*}{$(0.5 ; 0.3)$} & \multirow[t]{2}{*}{ EM } & BIAS & 0.0124 & 0.0230 & -0.0721 & 0.0471 & -0.2617 & -0.0021 & 0.0389 & -0.0193 & 0.0660 & -0.0142 & -0.0003 & 0.0106 \\
\hline & & & MSE & 0.0110 & 0.0295 & 0.3163 & 0.0488 & 0.7745 & 0.2417 & 0.0486 & 0.0323 & 0.0420 & 0.0040 & 0.0050 & 0.0036 \\
\hline & & \multirow[t]{2}{*}{ CEM } & BIAS & 0.1473 & 0.3125 & -0.6847 & 0.9754 & -0.5000 & -0.5020 & 0.2140 & -0.5300 & 0.3027 & -0.0041 & -0.0957 & 0.1013 \\
\hline & & & MSE & 0.1195 & 0.5900 & 2.8405 & 1.4259 & 1.9773 & 0.7873 & 0.0941 & 0.4811 & 0.2375 & 0.0339 & 0.0628 & 0.0505 \\
\hline & & \multirow[t]{2}{*}{ SEM } & BIAS & -0.0110 & 0.0280 & 0.0529 & -0.0573 & -0.1073 & -0.0010 & -0.0271 & -0.0771 & -0.0062 & -0.0071 & 0.0001 & 0.0047 \\
\hline & & & MSE & 0.0114 & 0.0291 & 0.2648 & 0.1345 & 0.3508 & 0.0859 & 0.0082 & 0.0263 & 0.0124 & 0.0035 & 0.0028 & 0.0028 \\
\hline
\end{tabular}



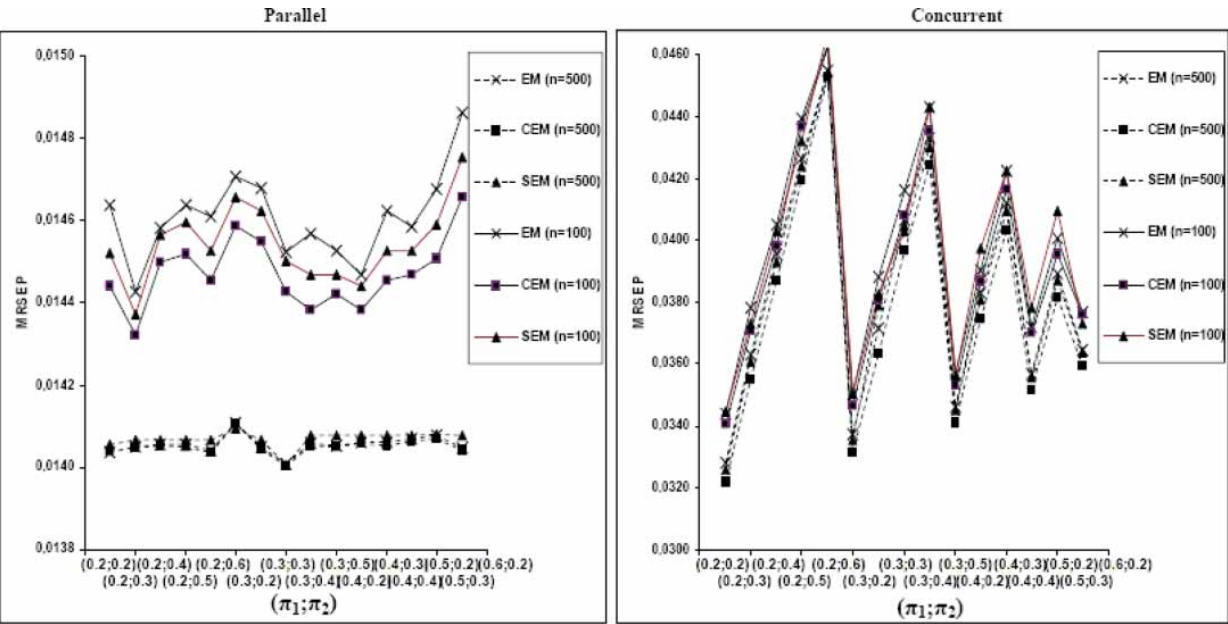

Figure 5. MRSEP by 10-fold cross-validation for three-component models when the true values were used as the starting values.
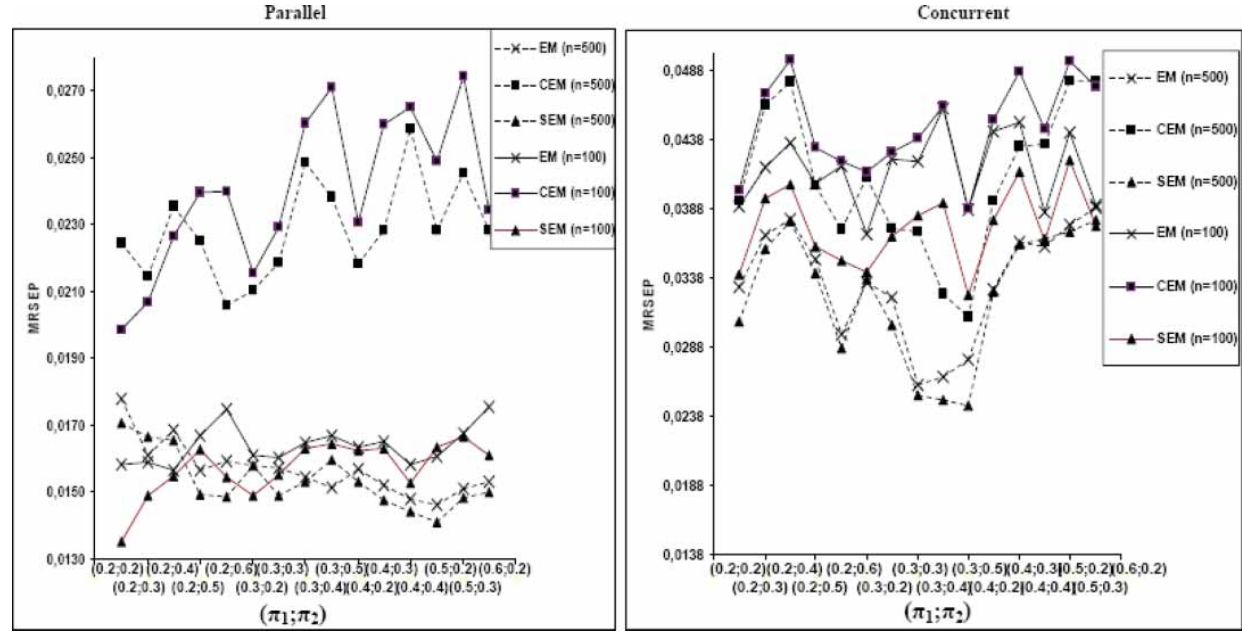

Figure 6. MRSEP by 10-fold cross-validation for three-component models when the algorithms were initiated by random numbers (second strategy).

\section{Conclusions and discussion}

In this article, we compare the performance of three algorithms to compute maximum likelihood estimates for the parameters of a mixture of linear regressions, the EM algorithm, the CEM algorithm and the SEM algorithm.

In our simulation study, we may conclude that CEM algorithm always converge in fewer iterations than the EM algorithm, which implies a reduction in the computational time to reach the parameter estimates.

When the true values are used as the starting values, the CEM algorithm applied to estimate the parameters of a mixture of linear regression provides, in general, best estimates in the sense of lower MSE. Also, through the $K$-fold cross-validation we can say that the CEM algorithm resulted in model estimates that best fit the regression model. 
When we run the algorithms from random initial position, in generality, the SEM algorithm outperforms the CEM and the EM algorithms by producing estimates of the parameters that have smaller MSE. Also, through the $K$-fold cross-validation we can say that the SEM algorithm resulted in model estimates that best fit the regression model.

\section{References}

[1] B.G.Lindsay, Mixture Models: Theory, Geometry and Applications, NSF-CMBS Regional Conference Series in Probability and Statistics, Vol. 5, Institute of Mathematical Statistics, Hayward, 1995.

[2] D. Böhning, Computer-Assisted Analysis of Mixtures and Applications: Meta-analysis, Disease Mapping and Others, Chapman \& Hall, New York, 2000.

[3] G.J. McLachlan and D. Peel, Finite Mixture Models, Wiley, New York, 2000.

[4] R. Quandt and J. Ramsey, Estimating mixtures of normal distributions and switching regression, J. Amer. Statist. Assoc. 73 (1978), pp. 730-738.

[5] R.D. De Veaux, Mixtures of linear regressions, Comput. Statist. Data Anal. 8 (1989), pp. 227-245.

[6] P.N. Jones and G.J. McLachlan, Fitting finite mixture models in a regression context, Aust. J. Statist. 34 (1992), pp. 233-240.

[7] T.R. Turner, Estimating the propagation rate of a viral infection of potato plants via mixtures of regressions, Appl. Statist. 49(3) (2000), pp. 371-384.

[8] D.S. Hawkins, D.M. Allen, and A.J. Stomber, Determining the number of components in mixtures of linear models, Comput. Statist. Data Anal. 38 (2001), pp. 15-48.

[9] H. Zhu and H. Zhang, Hypothesis testing in mixture regression models, J. Roy. Statist. Soc. 66(1) (2004), pp. 3-16.

[10] A.P. Dempster, N.M. Laird, and D.B. Rubin, Maximum likelihood from incomplete data via the EM algorithm, J. Roy. Statist. Soc. B 39 (1977), pp. 1-38.

[11] G. Celeux and G. Govaert, A classification EM algorithm and two stochastic versions, Comput. Statist. Data Anal. 14 (1992), pp. 315-332.

[12] G. Celeux and J. Diebolt, The SEM algorithm: a probabilistic teacher algorithm derived from the EM algorithm for the mixture problem, Comput. Statist. Quart. 2 (1985), pp. 73-82.

[13] S. Ganesalingam, Classification and mixture approach to clustering via maximum likelihood, Appl. Statist. 38 (1989), pp. $455-466$.

[14] G. Celeux and G. Govaert, Comparison of the mixture and the classification maximum likelihood in cluster analysis, J. Statist. Comput. Simul. 47 (1993), pp. 127-146.

[15] G. Govaert and M. Nadif, Comparison of the mixture and the classification maximum likelihood in cluster analysis with binary data, Comput. Statist. Data Anal. 23 (1993), pp. 65-81.

[16] G. Celeux, D. Chauveau, and J. Diebolt, Stochastic versions of the EM algorithm: an experimental study in the mixture case, J. Statist. Comput. Simul. 55 (1996), pp. 287-314.

[17] J. Dias and M. Wedel, An empirical comparison of EM, SEM and MCMC performance for problematic Gaussian mixture likelihoods, Statist. Comput. 14 (2004), pp. 323-332.

[18] R Development Core Team, $R$ : A language and environment for statistical computing, R Foundation for Statistical Computing, Vienna, Austria, 2008; software available at http://www.R-project.org.

[19] R.J. Hathaway and J.C. Bezdek, Switching regression models and fuzzy clustering, IEEE Trans. Fuzzy Syst. 1 (1993), pp. 195-204.

[20] T. Hastie, R. Tibshirani, and J. Friedman, Elements of Statistical Learning, Springer, New York, 2001. 